\title{
Superconducting vortex state in a mesoscopic disk containing a blind hole
}

\author{
G. R. Berdiyorov,* M. V. Milošević, B. J. Baelus, ${ }^{\dagger}$ and F. M. Peeters $\$$ \\ Departement Natuurkunde, Universiteit Antwerpen (Campus Drie Eiken), Universiteitsplein 1, B-2610 Antwerpen, Belgium
}

(Received 18 March 2004; published 19 July 2004)

\begin{abstract}
Within the phenomenological nonlinear Ginzburg-Landau theory, we studied the superconducting state of a mesoscopic superconducting disk with a circular blind hole. The influence of the smoothness of the blind hole edge, the thickness and size of the blind hole on the superconducting condensate and the vortex state is examined. We found that the presence of the blind hole in the superconductor increases the superconducting/ normal transition field. For large radii of the blind hole the maximal number of vortices that can nucleate in the sample increases with decreasing thickness of the blind hole. Vortices are preferentially captured in the blind hole and for a sufficiently large radius of the blind hole, the multivortex structure becomes energetically favorable. A gradual transition from a multivortex to a giant vortex state is observed.
\end{abstract}

DOI: 10.1103/PhysRevB.70.024508

PACS number(s): 74.20.De, 74.25.Dw, 74.78.Na, 74.25.Ha

\section{INTRODUCTION}

The properties of mesoscopic superconductors are very different compared to those of bulk superconductors as the vortex configurations and the critical parameters for mesoscopic samples are strongly influenced by the size and topology (boundary) of the samples. Because of their simple geometry, mesoscopic disks have been the most popular study objects during the last decade. Superconducting disks made of different materials were studied both experimentally and theoretically (see, for example, Refs. 1-4). As function of an applied external magnetic field, the disks showed various phase transitions within the superconducting state and depending on the sample dimensions and temperature, the superconducting-to-normal $(S / N)$ state transition could be of first-or second-order. Geim et al. studied also the paramagnetic Meissner effect in small superconductors ${ }^{5}$ and the nonquantized penetration of magnetic field in the vortex state of superconductors, ${ }^{6,7}$ which lead to fractional and negative vortices. Schweigert and Peeters ${ }^{8}$ discussed the phase transitions between different superconducting states and between the $S / N$ state of mesoscopic disks by solving numerically the Ginzburg-Landau (GL) equations. It was found that the type of phase transition depends on the disk size, namely, both the radius and the thickness. Furthermore, an analytical approach was developed to predict the type of phase transitions and to find the system characteristics near the phase transition point. In the continuation of their work ${ }^{9,10}$ (see also Ref. 11) transitions between different vortex configurations were predicted (splitting of the giant vortex into a multivortex, and vice versa).

Recently, due to the importance of pinning phenomena, the interest shifted to superconducting disks with a hole (antidot). Four decades ago, the limiting case-the thin-wire loop-was studied by Little and Parks. ${ }^{12}$ The $H-T$ phase diagram showed an oscillatory behavior each time an additional flux quantum $\Phi_{0}=h c / 2 e$ penetrated the sample. To describe the Little-Parks effect, Berger and Rubinstein ${ }^{13}$ studied nonuniform mesoscopic superconducting loops using the nonlinear GL theory. They assumed that the induced magnetic field can be neglected for samples with sufficiently small thickness. Superconducting ring structures were also studied by Baelus, Peeters, and Schweigert ${ }^{14}$ who found that the presence of the hole in the superconductor strongly influences the superconducting state. By increasing the size of the hole for the fixed radius of the disk, an enhanced stabilization of the superconducting state was found and more vortices can enter the sample. Transitions to a multivortex state were found for sufficiently large disks. Breaking the circular symmetry through a non central location of the hole in the disk favored the multivortex state in the superconductor. However, a direct experimental observation of the vortex structure in such mesoscopic samples is still lacking.

Many experiments ${ }^{15-17}$ were performed recently in order to study the vortex state in superconductors using magnetoresistance measurements. By measuring the resistivity of the non-fully-superconducting state one can obtain information about the $S / N$ transition boundary. With this technique it is possible to investigate the dependence of the critical parameters on the sample geometry, but it does not provide clear information about the real vortex structure. To describe these experimental results one can linearize the GL equations, simplifying the problem considerably. Another method to investigate the superconducting state is through Hall magnetometery, ${ }^{2,5,6,18}$ which gives indirect information on the vortex structure deep inside the superconducting region. The static and dynamic behavior of individual vortices can also be directly observed using the Bitter decoration technique, ${ }^{19,20}$ electron holography, ${ }^{21}$ scanning probe, ${ }^{22}$ and Lorent $\mathrm{z}^{23}$ microscopy. In the case of superconductors with holes it is impossible to visualize the vortices in the cavity because of the absence of any magnetic contrast. This can be circumvented by putting a thin superconducting layer under the sample (or equivalently by depositing a thin superconducting film inside the hole) and due to the so-called flux compression method, ${ }^{20}$ multiquanta vortices are visualized.

In the present paper we investigate nucleation of superconductivity in a superconducting disk containing a blind hole in the center using the full nonlinear GL theory. The present blind hole problem is a more general problem than the perforated superconductor, which we studied previously. ${ }^{14}$ Recently authors of Ref. 24 studied superconducting samples with periodic arrays of blind holes. To show 

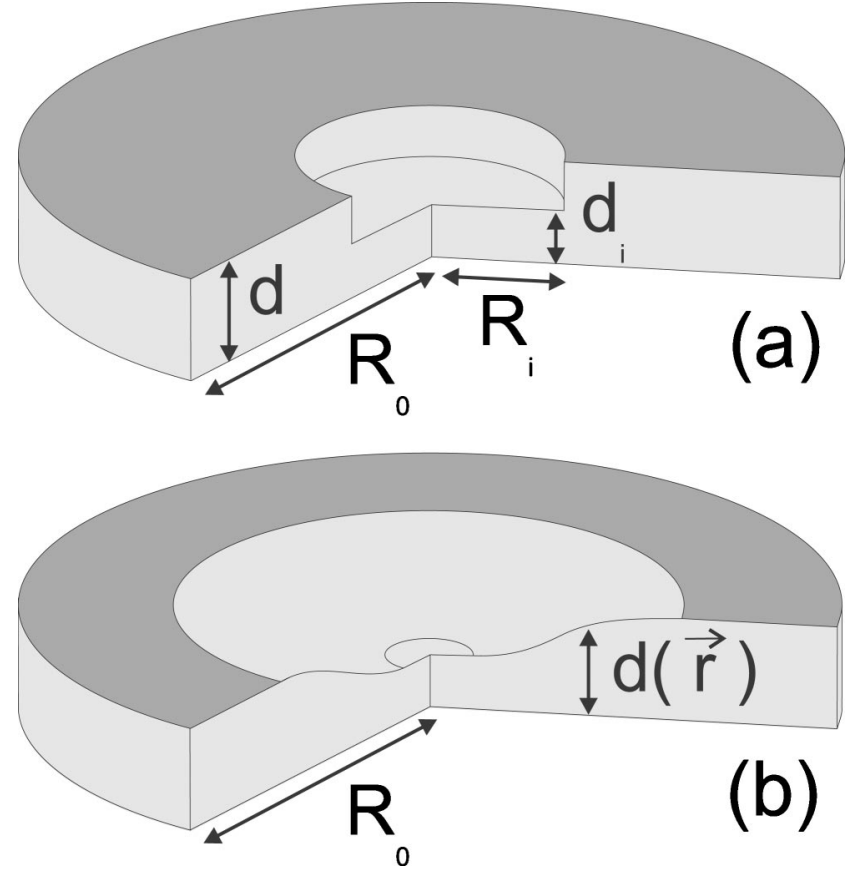

FIG. 1. The configurations: a superconducting disk with radius $R_{0}$ and thickness $d$ with (a) a sharp edge or (b) a smooth blind hole with radius $R_{i}$ and thickness $d(x, y)$, which is placed in the center of the disk.

the influence of the bottom superconducting layer on the critical field, Bezryadin, Ovchinnikov, and Pannetier studied a thin superconducting film with a blind circular hole, using the linearized GL equations. ${ }^{25}$ They found that the value of the critical field is sensitive to the bottom layer thickness, but the number of vortices, which nucleate inside the hole, was not influenced. Buzdin and Daumens considered similar structures using the analogy with electrostatic problems. ${ }^{26} \mathrm{In}$ our approach, the full nonlinear GL-theory is used and the dependence of various quantities (free energy, order parameter, and magnetization) on the thickness of the blind hole is studied. This allows us to obtain the actual vortex structure inside the blind hole, which will act as a pinning site when embedded in an infinite-extended superconducting film. We also consider the influence of the smoothness of the edges of the blind hole on the superconducting state. In two limiting cases (see Fig. 1): (i) $d=d_{i}$, i.e., for the superconducting disk, ${ }^{8,9}$ and (ii) $d_{i}=0$, the case of rings, ${ }^{14}$ we recover our previous results.

This paper is organized as follows. The theoretical formulation of the problem is presented in Sec. II, where we extend our previous approach to systems with variable thickness. The influence of the steepness of the edges of the blind hole on the vortex configuration and critical parameters is studied in Sec. III. In Secs. IV (small disks) and V (larger disks) we investigate the dependence of the superconducting state on the size of the disk and the blind hole, and on the thickness of the blind hole. The nonsymmetrical case, when the blind hole is moved from the center of the sample over a distance $a$ is considered in Sec. VI. The $H-T$ phase diagram is given in Sec. VII and our results are summarized in Sec. VIII.

\section{THEORETICAL FORMALISM}

In this work, we consider a superconducting disk with radius $R_{0}$ and thickness $d$ with a blind hole in the center with radius $R_{i}$ and thickness $d_{i}$ (see Fig. 1). The superconducting sample is immersed in an insulating medium (e.g., vacuum) and exposed to a homogeneous perpendicular magnetic field $\vec{H}_{0}=\left(0,0, H_{0}\right)$. In our calculation the demagnetization effects are taken into account and as an example we took the GL parameter $\kappa=1.0$, which is close to the experimental value for $\mathrm{Al}, \mathrm{Nb}$, or $\mathrm{Pb}$ mesoscopic samples. First we derive the GL equations for a thin sample with variable thickness $d(x, y)$. The total Gibbs free energy is ${ }^{27}$

$$
\begin{aligned}
G_{s H}= & G_{n H}+\int\left\{\alpha|\Psi|^{2}+\frac{1}{2} \beta|\Psi|^{4}+\frac{1}{4 m}\left|\left(-i \hbar \vec{\nabla}-\frac{2 e}{c} \vec{A}\right) \Psi\right|^{2}\right. \\
& \left.+\frac{1}{8 \pi}\left(\vec{H}-\vec{H}_{0}\right)^{2}\right\} d V,
\end{aligned}
$$

where $H$ is the local magnetic field and $G_{n H}$ is the free energy of the magnetic-field-exposed superconductor in the normal state. For thin disks we are allowed to assume that the superconducting condensate is homogeneous along the $z$-direction and consequently we may take for the volume of the sample $d V=d(x, y) d x d y$. To derive the GL equations we use the Euler equation:

$$
\frac{\partial \Omega}{\partial \Psi^{*}}-\frac{\partial}{\partial x} \frac{\partial \Omega}{\partial\left(\frac{\partial \Psi^{*}}{\partial x}\right)}-\frac{\partial}{\partial y} \frac{\partial \Omega}{\partial\left(\frac{\partial \Psi^{*}}{\partial y}\right)}=0,
$$

where $\Omega$ is the integrand appearing in Eq. (1). After simple transformations we obtain the expression

$$
\begin{aligned}
{[\alpha \Psi} & \left.+\beta \Psi|\Psi|^{2}+\frac{1}{4 m}\left(-i \hbar \vec{\nabla}-\frac{2 e}{c} \vec{A}\right)^{2} \Psi\right] d(x, y) \\
& -\frac{i \hbar}{4 m}\left(-i \hbar \vec{\nabla} \Psi-\frac{2 e}{c} \vec{A} \Psi\right) \vec{\nabla} d(x, y) \\
& +\frac{i \hbar}{4 m} d(x, y) \vec{\nabla}\left(i \hbar \vec{\nabla} \Psi+\frac{2 e}{c} \vec{A} \Psi\right)=0,
\end{aligned}
$$

which gives us the boundary condition $\vec{n}(-i \hbar \vec{\nabla}$ $-2 e / c \vec{A})\left.\Psi\right|_{\text {boundary }}=0$ and the first GL equation (see also Ref. 28):

$$
\begin{aligned}
& \frac{1}{2 m}\left(-i \hbar \vec{\nabla}-\frac{2 e}{c} \vec{A}\right)^{2} \Psi \\
& \quad=-\alpha \Psi-\beta \Psi|\Psi|^{2}+\frac{i \hbar}{2 m}\left(-i \hbar \vec{\nabla}-\frac{2 e}{c} \vec{A}\right) \Psi \frac{\vec{\nabla} d(x, y)}{d(x, y)},
\end{aligned}
$$

where $d(x, y)$ is the coordinate-dependent thickness of our sample. The last term in Eq. (4) describes the effect of the sample thickness variation on the superconducting condensate. In case of a sharp hole with radius $R_{i}$ the latter term becomes a delta function, which results in a discontinuity of the derivative of the order parameter at $\rho=R_{i}$, while the order 


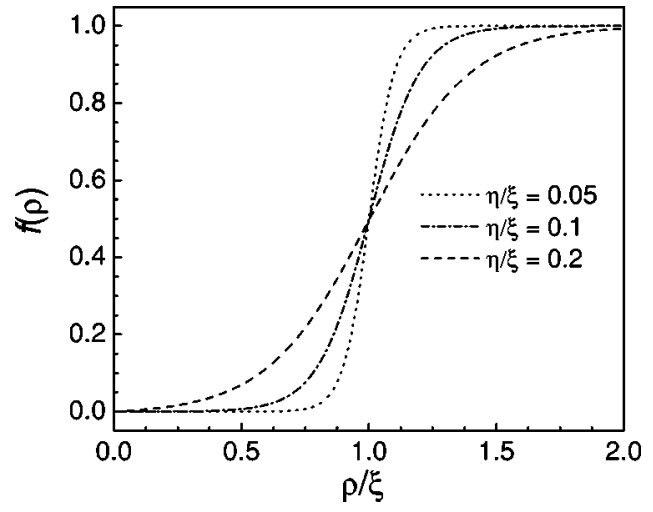

FIG. 2. The function $f(\rho)=[1-\exp (-\rho / \eta)] /\left[1+\exp \left(0.5 R_{0}\right.\right.$ $-\rho) / \eta]$ describing the smoothness of the blind hole edge for different values of the parameter $\eta / \xi$.

parameter itself is continuous. Thus at the edge $\rho=R_{i}$ the radial component of the current density $\vec{j}$ will exhibit a jump, but the total current $\vec{I}=\vec{j} d$ has to be continuous.

The second GL equation reads:

$$
\vec{\nabla} \times \vec{\nabla} \times \vec{A}=\frac{4 \pi}{c} \vec{j}
$$

with the superconducting current density

$$
\vec{j}=\frac{e \hbar}{i m}\left(\Psi^{*} \vec{\nabla} \Psi-\Psi \vec{\nabla} \Psi^{*}\right)-\frac{4 e^{2}}{m c}|\Psi|^{2} \vec{A} .
$$

The actual boundary condition corresponds to the preservation of the total current in the perpendicular cross section of the sample, which can be written as the condition of no current leaking in the insulator media $\left.(-i \vec{\nabla}-\vec{A}) \Psi\right|_{n}=0$, where the subscript $n$ denotes the component normal to the disk surface. The boundary condition for the vector potential has to be taken far away from the disk, where $H$ equals the applied field, i.e., $\vec{A}=\vec{A}_{0}=0.5 H_{0} \rho \vec{e}_{\phi}$ for $\rho \gg R_{0}$. Here $\vec{e}_{\phi}$ denotes the azimuthal direction, and $\rho$ the radial distance from the disk center.

In this paper we consider thin superconducting samples $\left(d, d_{i} \ll \xi, \lambda\right)$ and in this case the GL equations may be averaged over the thickness of the superconductor. Using dimensionless variables and the Landau gauge, $\operatorname{div} \vec{A}=0$, we rewrite the system of coupled nonlinear Eqs. (4) and (5) in the following form:

$$
\begin{gathered}
(-i \vec{\nabla}-\vec{A})^{2} \Psi=\Psi\left(1-|\Psi|^{2}\right)+i(-i \vec{\nabla}-\vec{A}) \Psi \frac{\vec{\nabla} d(x, y)}{d(x, y)}, \\
-\frac{\kappa^{2}}{d(x, y)} \Delta \vec{A}=\frac{1}{2 i}\left(\Psi^{*} \vec{\nabla} \Psi-\Psi \vec{\nabla} \Psi^{*}\right)-|\Psi|^{2} \vec{A}
\end{gathered}
$$

and we solve this system by following the numerical approach of Schweigert and Peeters. ${ }^{8}$

Here all distances are measured in units of the coherence length $\xi=\hbar / \sqrt{-2 m \alpha}$, the order parameter in $\psi_{0}=\sqrt{-\alpha / \beta}$, and the vector potential in $c \hbar / 2 e \xi . \kappa=\lambda / \xi$ is the GL parameter, and $\lambda=c \sqrt{m / \pi} / 4 e \psi_{0}$ is the penetration depth. We scale the superconducting current in units of $j_{0}=c H_{c} / 2 \pi \xi$ and the magnetic field in $H_{c 2}=c \hbar / 2 e \xi^{2}=\kappa \sqrt{2} H_{c}$, where $H_{c}$ $=\sqrt{-4 \pi \alpha / \beta}$ is the critical field.

The difference between the superconducting and the normal state Gibbs free energy [Eq. (1)] measured in $F_{0}$ $=H_{c}^{2} V / 8 \pi$ units can be expressed through the integral

$$
\begin{aligned}
F= & V^{-1} \int_{V}\left[2\left(\vec{A}-\vec{A}_{0}\right) \vec{j}-|\Psi|^{4}\right. \\
& \left.+i(-i \vec{\nabla}-\vec{A}) \Psi \frac{\vec{\nabla} d(x, y)}{d(x, y)}\right] d(x, y) d x d y,
\end{aligned}
$$

where integration is performed over the sample volume $V$, and $\vec{A}_{0}$ is the vector potential of the applied uniform magnetic field. The dimensionless magnetization, which is a direct measure of the expelled magnetic field from the sample, is defined as

$$
M=\frac{\langle H\rangle-H_{0}}{4 \pi},
$$

where $\langle H\rangle$ denotes the magnetic field averaged over the sample.

For nonzero temperature $T$, the temperature dependence of $\xi$ and $H_{c 2}$ is given by

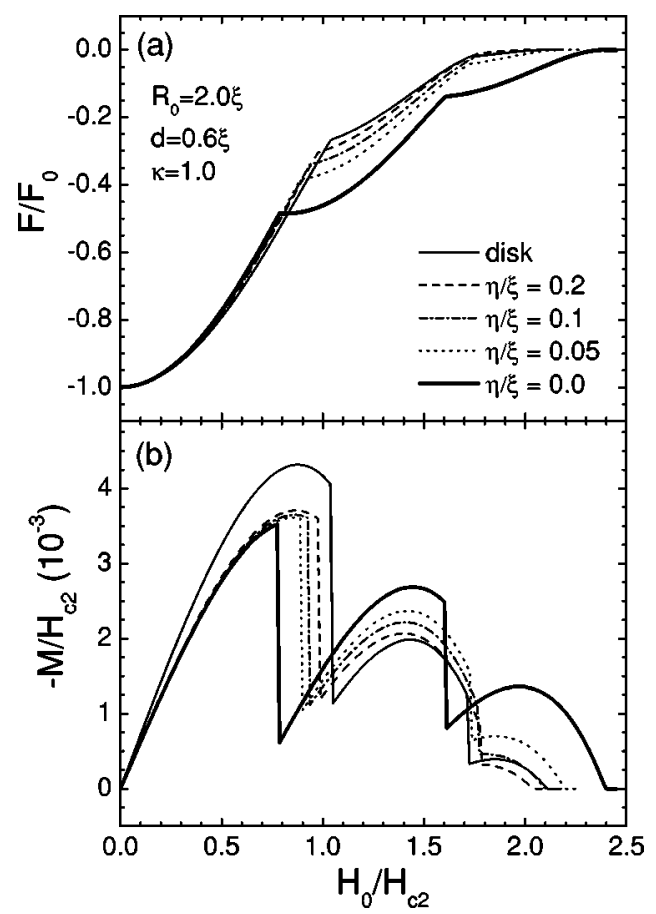

FIG. 3. The ground state free energy (a) and the corresponding magnetization (b) as a function of the applied magnetic field for a disk (thin solid curve) with radius $R_{0}=2.0 \xi$ and thickness $d=0.6 \xi$ and for samples containing a blind hole with different steepness: $\eta / \xi=0.2$ (dashed curve), 0.1 (dash-dotted curve), 0.05 (dotted curve), and 0.0 (thick solid curve). The GL parameter is $\kappa=1.0$. 


$$
\begin{gathered}
\xi(T)=\frac{\xi(0)}{\sqrt{\left|1-T / T_{c 0}\right|}}, \\
H_{c 2}(T)=H_{c 2}(0)\left|1-\frac{T}{T_{c 0}}\right|
\end{gathered}
$$

where $T_{c 0}$ is the critical temperature corresponding to the transition to the normal state at zero magnetic field. This scaling allows us to relate our numerical results to the experimental measurements performed at nonzero temperature.

\section{EFFECT OF THE STEEPNESS OF THE BLIND HOLE EDGE ON THE VORTEX STATE}

In this section we investigate the influence of the smoothness of the blind hole edges on the vortex configurations by changing the smoothness and the slope of the edges of the blind hole. In order to avoid Dirac-delta-functions in the differential equation (7), which appear in the case of a step-like change in $d(x, y)$, we introduced a gradual thickness variation, modelled by the function $f(\rho)=[1-\exp (-\rho / \eta)] /$ $\left[1+\exp \left(R_{i}-\rho\right) / \eta\right]$, which is plotted in Fig. 2, for $\eta / \xi$ $=0.05,0.1,0.2$ in the case of $R_{i}=R_{0} / 2=1.0 \xi$. In the limit $\eta$ $\rightarrow 0$ the function $f(\rho)$ reduces to the Heaviside step function. The thickness of our sample is defined as $d(\rho)=a+b \cdot f(\rho)$, where $a=d_{i}$ and $b=d-d_{i}$.

We consider a small superconducting sample with $a$ $=0.3 \xi, b=0.3 \xi, R_{i}=\xi, R_{0}=2.0 \xi$, and $\eta / \xi=0.0,0.05,0.1,0.2$. It was shown in Ref. 8 that for small radius disks the confinement effects are dominant and this imposes a circular symmetry on the superconducting condensate. Therefore, following the approach of Ref. 8 we solve the GL equations assuming $\Psi(\rho)=F(\rho) \exp (i L \phi)$, where $\rho$ and $\phi$ are cylindrical coordinates, and consequently both the vector potential and the superconducting current are directed along $\vec{e}_{\phi} . L$ is the winding number and gives the vorticity of the system. As we restricted ourselves to circular symmetric configurations, the present states characterize only the giant vortex states. In this case, the number of variables in the GL equations are reduced, which improves the accuracy and shortens the computational time.

Figs. 3(a) and 3(b) show the ground state free energy and magnetization for the above sample and for the case in the absence of a blind hole with disk thickness $d(=a+b)=0.6 \xi$. In all cases, only a maximum of two vortices can nucleate in the superconductor. The free energy of the Meissner state for the disk is lower than the energy of the other samples, which is a consequence of the enhanced penetration of the magnetic field into the superconductor when the blind hole is present [see Fig. 4(a)]. For the same reason, the thermodynamic transition field between the $L=0$ and $L=1$ states is higher for the disk. This field is the smallest for the $\eta / \xi=0.0$ case, i.e., the case of a perfect blind hole (steep edge). The free energy of the $L=1$ and $L=2$ states is lower for smaller $\eta / \xi$, which illustrates the compression of vortices into the center of the sample [see Fig. 4(b)]. For the $L=2$ state the value of the free energy of the disk is lower than the energy of the sample with $\eta / \xi=0.1$ and 0.2 , which results in a higher superconducting/normal $(S / N)$ transition field. This field is $H_{c 3} / H_{c 2}=2.11$ for the disk and $H_{c 3} / H_{c 2}=2.04,2.10,2.20$, 2.40 for the cases $\eta / \xi=0.2,0.1,0.05$, and 0.0 , respectively.

Following the pattern explained above, the magnetization $(-M)$ of the disk in the Meissner state is larger than for the
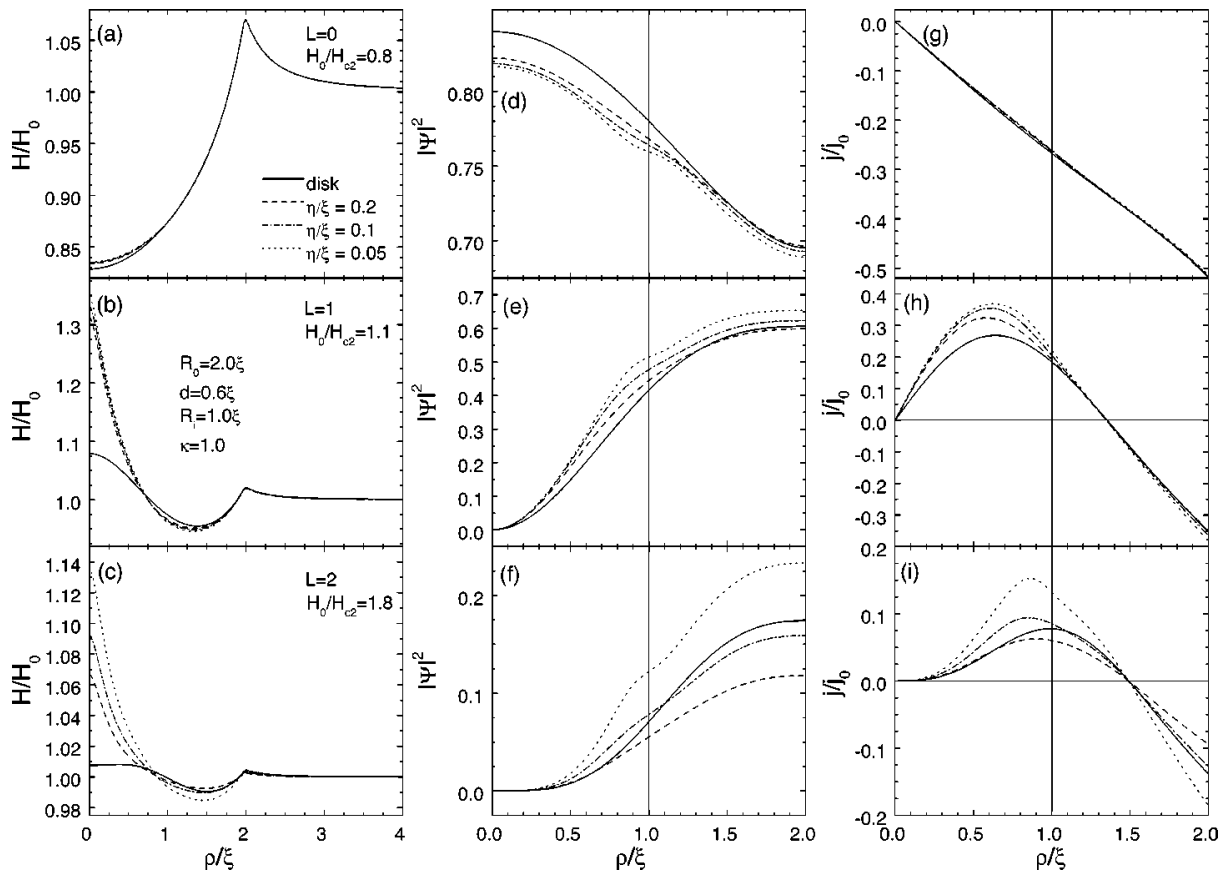

FIG. 4. The magnetic field (a)-(c), Cooper-pair density (d)-(f) and current density (g)-(i) distributions as function of the radial distance for the states with vorticity $L=0,1$, and 2 , at $H_{0} / H_{c 2}=0.8,1.1$, and 1.8 , respectively, for a superconducting disk (solid curve) with radius $R_{0}=2.0 \xi$ and thickness $d=0.6 \xi$ and for samples with a blind hole with radius $R_{i}=1.0 \xi$ and with different steepness characterized by the shape parameter $\eta / \xi=0.2$ (dashed curve), 0.1 (dash-dotted curve), and 0.05 (dotted curve). 
samples with a blind hole, which is due to the larger flux expulsion from the sample. For $L>0$ the relative position of the different magnetization curves depends on the magnetic field.

In what follows, we investigate the Cooper-pair density $|\Psi|^{2}$, the local magnetic field $H$, and current density distributions. Fig. 4 shows these quantities as a function of the radial distance $\rho$ for the states with $L=0(\mathrm{a}, \mathrm{d}, \mathrm{g}), 1(\mathrm{~b}, \mathrm{e}, \mathrm{h})$ and $2(\mathrm{c}, \mathrm{f}, \mathrm{i})$ at $H_{0} / H_{c 2}=0.8,1.1$, and 1.8 , respectively. At these values of the applied magnetic field the given vorticity corresponds to the ground state of the sample. The local magnetic field is scaled by the applied magnetic field and the current density is expressed in units of $j_{0}=c H_{c} / 2 \pi \xi$. In the Meissner state [Fig. 4(a)], the magnetic field distribution is the same for all samples, enhanced at the sample boundary (maximum) and the surrounding insulating medium. However, the magnetic field in the center of the disk is slightly lower than for the samples with an incavation. This is due to the induced supercurrent, which compensates the effect of the applied magnetic field on the superconductor. Therefore, the Cooper-pair density in the center is higher for the disk than for the other samples, while near the outer boundary $|\Psi|^{2}$ is higher for the sample with $\eta / \xi=0.2$ [Fig. 4(d)]. All the current in the superconducting disk is directed in the clockwise direction, with its maximum at the disk edge. Therefore, the applied magnetic field is actually enhanced close to the disk boundary, which leads to a stronger depression of the Cooper-pair density at the outer edges as compared to the central region of the sample [Fig. 4(d)]. Comparing the Meissner currents in the case of a "classical" disk and the disks with incavations, very little can be seen, as the current shows almost the same qualitative and quantitative behavior.

For the $L=1$ state [Fig. 4(b)] the flux is compressed in the center of the sample and the magnetic field even becomes larger than the applied field. In the central region the magnetic field is lower for the ones with a blind hole, while the field at the edge of the samples is the same in all cases. The Cooper-pair density of this state is higher for the sample with $\eta / \xi=0.05$ than for the other samples [Fig. 4(e)]. In this case the sign of the current in the central region of the superconductor becomes positive (the current direction reverses) due to the presence of the vortex, but the current near the outer boundary remains negative [Fig. 4(h)]. Close to the center the magnetic field is compressed into the superconductor (paramagnetic effect), while near the outer boundary the magnetic field is expelled to the insulating media (diamagnetic effect). The positive current near the center and the negative current near the edges are stronger for the sample with $\eta / \xi=0.05$ than in the classical disk case.

When the second vortex enters the superconductor [Fig. 4(c)], the magnetic field in the samples with lower $\eta$ is more enhanced (i.e., as we approach the perfect blind hole case) since the indentation in the center of the sample favors the capture of vortices in the center of the disk. The Cooper-pair density of the disk is higher at the disk boundary than for the samples with geometrical parameter $\eta / \xi=0.1$ and 0.2 (therefore, the free energy is lower), but in the central region of the disk the Cooper-pair density is lower than in the disks with an incavation [Fig. 4(f)]. The current distribution for the

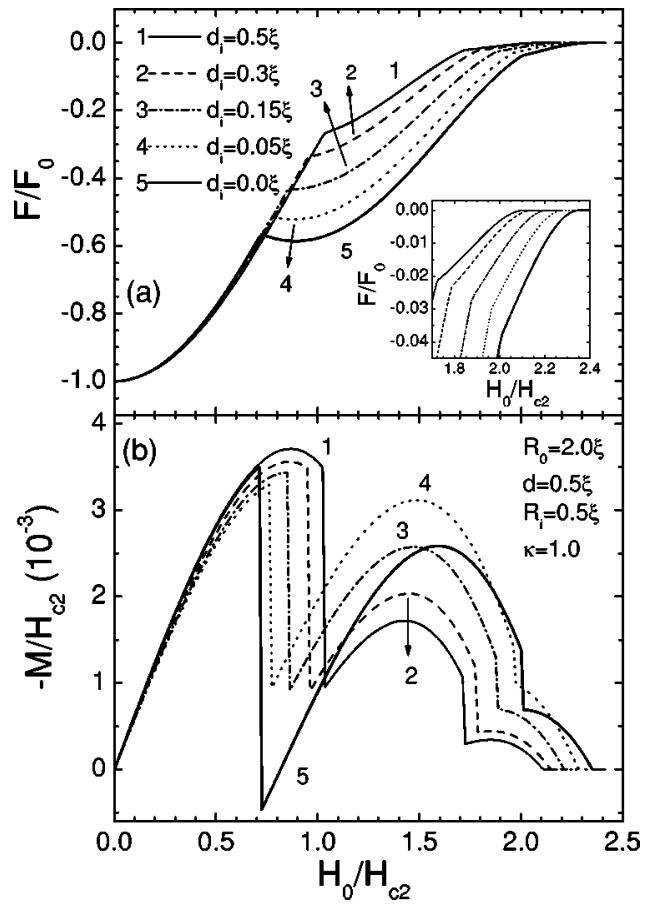

FIG. 5. The ground state free energy (a) and the corresponding magnetization (b) as a function of the applied magnetic field, for a disk (curve 1) with radius $R_{0}=2.0 \xi$ and thickness $d=0.5 \xi$, a disk with a blind hole in the center with radius $R_{i}=0.5 \xi$ and thickness $d_{i} / \xi=0.3$ (curve 2), 0.15 (curve 3), 0.05 (curve 4), and 0.0 (superconducting ring, curve 5). The inset in (a) is an enlargement of the high magnetic field region where the $L=2$ state is the ground state.

$L=2$ state is analogous to the $L=1$ state, but in this case the difference between the values of the current densities for the different samples is more pronounced [Fig. 4(i)].

With decreasing the geometrical parameter $\eta$, namely making the edges of the blind hole steeper, the change of curvature in the order parameter can be seen close to the blind hole boundary [see Figs. 4(d) -4 (f), for $\eta / \xi=0.05$ ]. Due to the continuous function $f(\rho)$ describing the shape of the superconducting disk in our analysis, both the order parameter and its derivative, with respect to $\rho$ are continuous. However, in the case of a perfect blind hole, a jump in the derivative of the order parameter should be present at the edge of the hole due to the preservation of the total current in every cross section of the disk (as will be shown in the following sections of this article), and the change of the curvature of the order parameter in Figs. 4(d)-4(f), for $\eta / \xi$ $=0.05$ is therefore a logical consequence of the steeper blind hole edges.

\section{SMALL SUPERCONDUCTING DISKS WITH A BLIND HOLE}

Using the theoretical approach of the previous section we consider small superconducting disks with a perfect blind hole in the center [see Fig. 1(a)], i.e., for $\eta / \xi=0$ and investigate the effect of the thickness of the blind hole on the superconducting state. Figs. 5(a) and 5(b) show the ground state free energy and the magnetization of such a supercon- 
ducting disk with radius $R_{0}=2.0 \xi$ and thickness $d=0.5 \xi$ with a blind hole with radius $R_{i}=0.5 \xi$ and thickness $d_{i} / \xi=0.0$, $0.05,0.15,0.3$, and 0.5 . The inset shows the enlargement of the free energy in the region where the $L=2$ state becomes the ground state. The situation with $d_{i}=0.5 \xi$ corresponds to the "classical" disk case ${ }^{8}$ and $d_{i}=0.0 \xi$ to the superconducting disk with a hole (superconducting ring). ${ }^{14}$ In all cases the maximal possible vorticity in the sample equals $L_{\max }=2$. When we decrease the blind hole thickness the Meissner state, i.e. the $L=0$ state becomes less stable and the $L=0$ $\rightarrow L=1$ state transition occurs at lower magnetic field [which is similar to the previous continuous edge case, where decreasing $\eta / \xi$ implies a decreasing effective thickness of the blind hole, see Fig. 3(b)]. Also the ground state free energies for the $L=1$ and $L=2$ states are lower for the samples with small thickness of the blind hole. Notice that the $L=1 \rightarrow 2$ transition occurs at higher fields with decreasing $d_{i}$ [see inset of Fig. 5(a)], which is opposite to the $L=0 \rightarrow 1$ transition and is also different in the case of the continuous edge. With decreasing the thickness of the blind hole the $S / N$ state transition shifts to higher magnetic fields. This field is $H_{c 3} / H_{c 2}$ $=2.11$ for the disk, $H_{c 3} / H_{c 2}=2.35$ for the ring and $H_{c 3} / H_{c 2}$ $=2.15,2.21$, and 2.29 for a thickness of the blind hole $d_{i} / \xi$ $=0.3,0.15$, and 0.05 , respectively.

The magnetization, $-M$, [Fig. 5(b)] of the $L=0$ state is higher for the disk than for the other samples, which shows the enhanced expulsion of the field from the disk. However, for the $L=1$ state the magnetization of the disk is smaller, since the presence of the blind hole in the center favors the appearance of vortices (compression of the flux in the center of the sample). With decreasing the blind hole thickness the magnetization of this state increases. The ground state of the

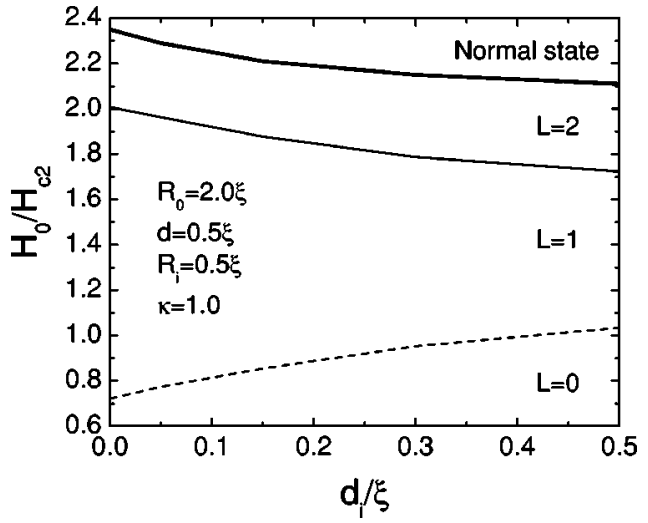

FIG. 6. Phase diagram: the relation between the blind hole thickness $d_{i}$ and the magnetic field $H_{0} / H_{c 2}$ at which ground state transitions take place for a superconducting sample with radius $R_{0}$ $=2.0 \xi$, thickness $d=0.5 \xi, \kappa=1.0$ and with blind hole radius $R_{i}$ $=0.5 \xi$.

ring with $L=1$ shows a paramagnetic response, i.e., $-M<0$. For the other samples this effect occurs only for the metastable states with $L=1$ and $L=2$.

Figure 6 summarizes these results into a phase diagram which gives the relation between the blind hole thickness $d_{i}$ and the magnetic field $H_{0} / H_{c 2}$ at which the ground state transitions take place for a superconducting disk with radius $R_{0}=2.0 \xi$, thickness $d=0.5 \xi, \kappa=1.0$, and a blind hole with radius $R_{i}=0.5 \xi$. The dashed curve indicates the ground state transition from the $L=0$ state to the $L=1$ state, the thin solid curve the $L=1$ to the $L=2$ state transition, and the thicker solid curve gives the $S / N$ transition. Notice that the Meissner state is stabilized as being the ground state with increasing
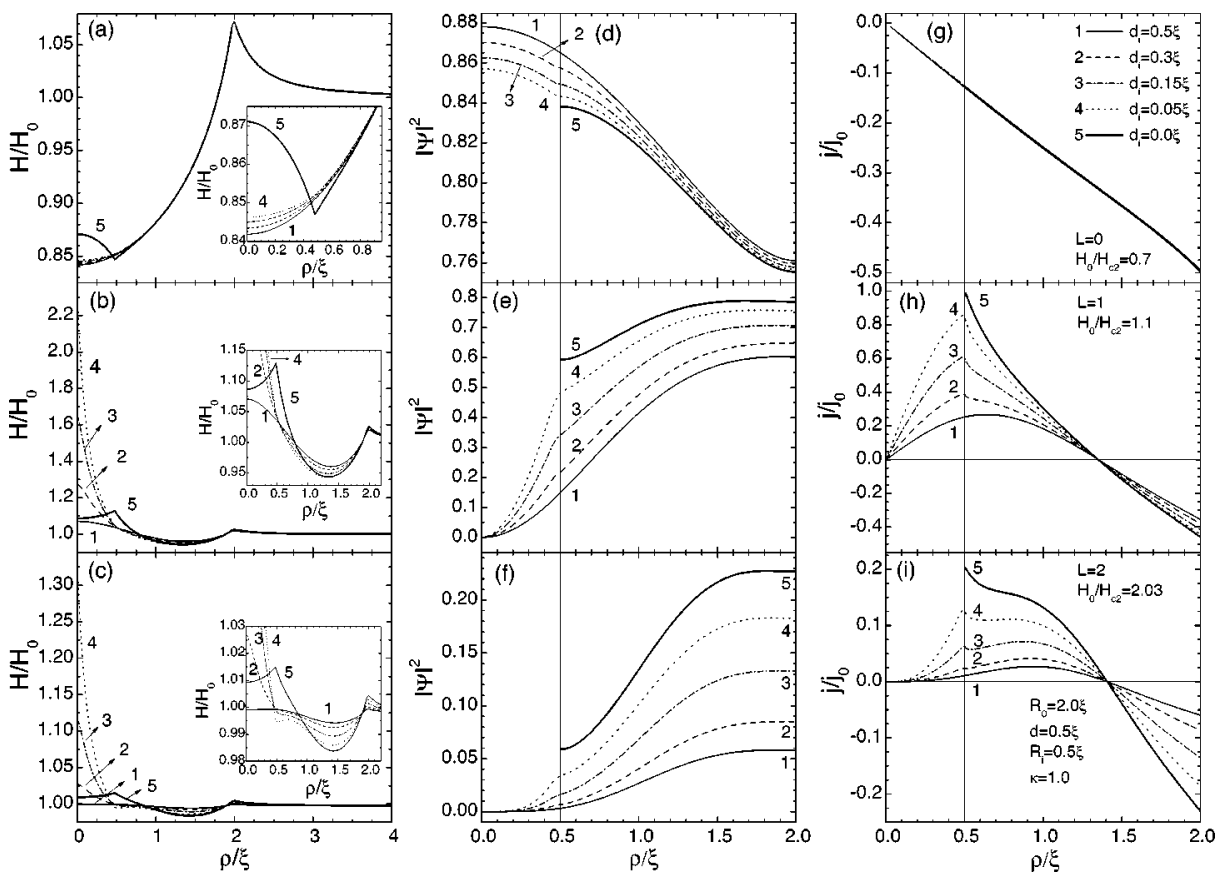

FIG. 7. The magnetic field (a)-(c), Cooper-pair density (d)-(f), and current density (g)-(i) distributions as a function of the radial distance for the superconducting states with $L=0(\mathrm{a}, \mathrm{d}, \mathrm{g}), 1(\mathrm{~b}, \mathrm{e}, \mathrm{h})$, and $2(\mathrm{c}, \mathrm{f}, \mathrm{i})$ at $H_{0} / H_{c 2}=0.75,1.55$, and 2.0, respectively, for the disk (curve 1) with radius $R_{0}=2.0 \xi$ and thickness $d=0.5 \xi$, with a blind hole in the center with radius $R_{i}=0.5 \xi$ and thickness $d_{i} / \xi=0.3$ (curve 2), 0.15 (curve 3), 0.05 (curve 4), and 0.0 (superconducting ring, curve 5). The insets show the magnetic field near the boundary of the blind hole. 


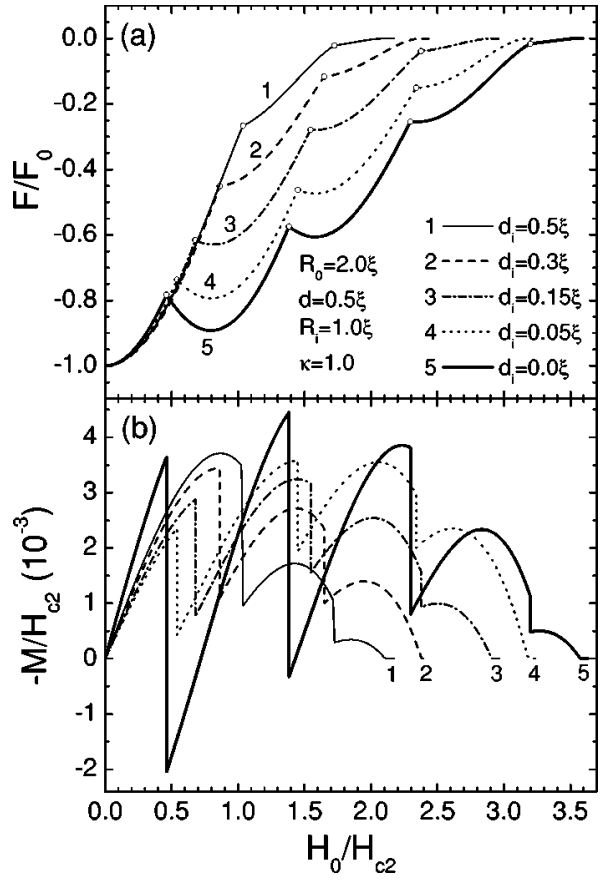

FIG. 8. The ground state free energy (a) and corresponding magnetization (b) as a function of the applied magnetic field of a superconducting disk with the same parameters as in Fig. 7 (curves $1-5$ ), except for the radius of the blind hole $R_{i}=1.0 \xi$. Open circles indicate transitions between different vortex states.

thickness of the blind hole, which is opposite to the $L=1$ $\rightarrow L=2$, and the $S / N$ state transition field, which moves to smaller fields with increasing $d_{i}$.

Figure 7 shows similar results as Fig. 4, but now for a smaller radius blind hole, i.e., $R_{i}=0.5 \xi$ instead of $R_{i}=1.0 \xi$ and where we study the dependence on the thickness of a steplike (i.e., $\eta=0$ ) blind hole. For the $L=0$ state the magnetic field distribution for the disk and for the samples with a blind hole is almost the same at the outer edges and outside of the sample Fig. 7(a). The local magnetic field is lower than the external magnetic field inside the sample and it increases at the outer edge of the sample, where the field lines are compressed. Due to the weak penetration of the magnetic field inside the superconductor the Cooper-pair density is lower at the outer edges of the samples [Fig. 7(d)]. As one can see in the inset of [Fig. 7(a)] the magnetic field in the center of the samples with a blind hole is higher than in the disk case and therefore the highest value of the Cooper-pair density is found in the case of a "classical" disk. With decreasing thickness of the blind hole this value decreases and approaches the value of the density of the ring. For the sample with a hole, a different behavior is observed and the magnetic field increases in the center since the screening currents expel part of the applied magnetic field toward the inside of the hole as well as toward the outside of the disk.

The magnetic field distribution is considerably changed when the first vortex enters the sample [Fig. 7(b)]. In this case, because of the demagnetization effect, in the ring case there is a sharp peak in the magnetic field at the inner boundary and the magnetic field near the center of the sample is higher than the external magnetic field. However, for the samples with the blind hole no peak at the inner hole boundary is present, and the magnetic field is increased in the center of the sample. Consequently, the probability of finding superconducting electrons inside the disk is smaller than if the blind hole is present. With decreasing thickness of the blind hole the value of the magnetic field in the center becomes higher and the Cooper-pair density increases near the boundaries [Fig. 7(e)]. The magnetic field distribution at the outer boundary of the sample is similar for all samples. The Cooper-pair density increases abruptly when the thickness of the blind hole is small, showing the enhanced compression of the magnetic flux in the blind hole.

The magnetic field distribution and the Cooper-pair density for the $L=2$ state is analogous to the field distribution and $|\Psi|^{2}$ for the $L=1$ state [Figs. 7(c) and 7(f)].

One of the most interesting features is the jump in the derivative of the order parameter over the radial distance at the blind hole edge. Due to the condition of the continuity of the order parameter and the shape of the thickness of our sample, which is actually a step function, the jump in $\partial \Psi / \partial \rho$ is a consequence of the last term in Eq. (7).

The qualitative behavior of the current density [Figs. 7(g)-7(i)] corresponds to the one of Fig. 4. The current density of the $L=0$ state is almost the same for all samples, regardless of the thickness of the blind hole. As the current has only a $j_{\varphi}$ component there is no jump in the current density at the edge of the blind hole, but the total current has a jump. For the $L=1$ and $L=2$ states the current near the blind hole boundary is positive and near the outer boundary is negative, and the amplitudes increase with decreasing thickness of the bottom of the blind hole.

It was shown in Ref. 14 that with increasing inner radius of the superconducting ring the $S / N$ transition field shifts to higher magnetic fields and more transitions between different vortex states are possible. In Figs. 8(a) and 8(b) the ground state free energy and the corresponding magnetization of a superconducting disk with a larger blind hole $\left(R_{i}=1.0 \xi\right)$ is shown while keeping the other parameters the same as before. In this case the maximal number of vortices for the sample with a blind hole of thickness $d_{i}=0.3 \xi$ is $L_{\max }=2$. For a thinner blind hole, more vortices can enter the sample before destroying the superconducting state. The maximal number of vortices for the ring is $L_{\max }=4$. By decreasing the

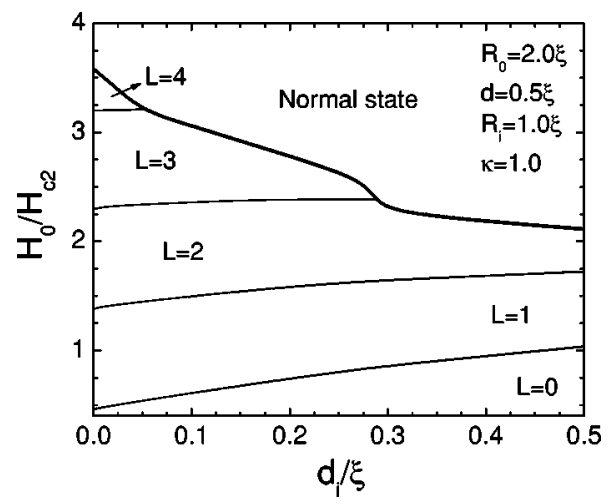

FIG. 9. The same as Fig. 6, but now for larger radius of the blind hole, $R_{i}=1.0 \xi$. 


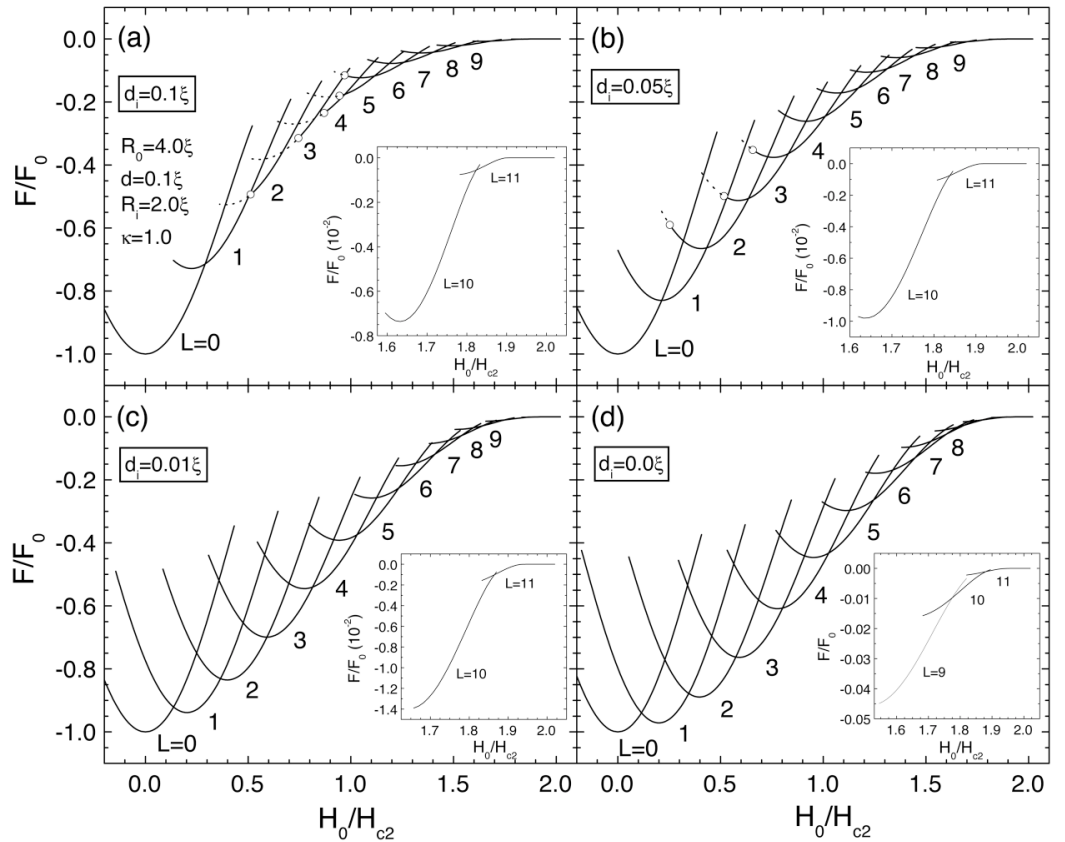

FIG. 10. The free energy as a function of the applied magnetic field of the superconducting disk with radius $R_{0}=4.0 \xi$, thickness $d$ $=0.1 \xi$ (a) with a blind hole in the center with radius $R_{i}=2.0 \xi$ and thickness $d_{i}=0.05 \xi(\mathrm{b}), 0.01 \xi$ (c), and 0 (superconducting ring) (d), respectively. The multivortex states are plotted by dotted curves and the transitions from the multivortex state to the giant vortex state are indicated by open circles. thickness of the blind hole, the $S / N$ transition field shifts to higher fields. This field is $H_{0} / H_{c 2}=2.11$ in the disk case and $H_{0} / H_{c 2}=2.38,2.92,3.21$ for the thickness of the blind hole $d_{i} / \xi=0.3,0.15,0.05$, respectively. The $S / N$ transition field for the ring equals $H_{0} / H_{c 2}=3.58$. The transitions between different $L$ states occur at lower fields for the small thickness of the blind hole, and the free energy becomes lower, approaching the free energy of the superconducting ring.

The phase diagram in Fig. 9 shows the magnetic field $H_{0} / H_{c 2}$, at which ground state transitions take place, as a function of $d_{i}$ for larger value of the $R_{i}$. The thick solid curve gives the $S / N$ transition, which exhibits some oscillatory-like behavior. Notice that the $S / N$ transition moves to higher field with decreasing the blind hole thickness and in that case more vortices can be trapped. Also that the phase diagram is very different from the $R_{i}=0.5 \xi$ case (Fig. 6) because now all ground state transition fields increase with $d_{i}$ except for the $S / N$ transition field.

\section{LARGER DISKS WITH A BLIND HOLE: MULTIVORTEX STATES}

Until now, we restricted ourselves to small superconducting samples, where the confinement effects are dominant and only giant vortex states are stable. For larger superconducting disks it is energetically more favorable for the giant vortex to split into separated vortices for certain magnetic fields. ${ }^{9}$

To investigate such multivortex states we generalize the approach of Ref. 9 to superconducting disks with a blind hole in the center. No special symmetry is imposed on the superconducting condensate $\Psi(x, y)$, which is allowed to be of arbitrary shape. As an example we take a superconducting disk with $\kappa=1.0$, radius $R_{0}=4.0 \xi$, thickness $d=0.1 \xi$, and for different values of the radius $R_{i}$ and thickness $d_{i}$ of the blind hole. Figures 10(a)-10(d) show the free energy for such a disk containing a blind hole with radius $R_{i}=2.0 \xi$ and thickness $d_{i}=0.1 \xi, 0.05 \xi, 0.01 \xi$, and 0 (superconducting ring), respectively, as a function of the applied magnetic field. The insets show an enlargement of the free energy close to the $S / N$ boundary. The multivortex states are plotted by dotted curves and the transitions from the multivortex state to the giant vortex state are indicated by open circles. In order to define whether the state is a multivortex state or a giant vortex state, we used the criterion of Ref. 29, which states that if the maximum between two minima in the Cooper-pair density is lower than $0.5 \%$ of the maximum Cooper-pair density in the sample, the state is assigned to be a giant vortex state. In all samples vortex states up to $L=11$ can nucleate (see also Fig. 11). In the case of the uniform disk multivortex states can nucleate for vorticity $L=2,3,4,5$, and 6 , and with increasing external field, these multivortex states transit to a giant vortex state for fixed $L$. When we include a blind hole into the disk [Fig. 10(b)] the free energy of all states becomes lower and the $S / N$ state transition occurs at higher

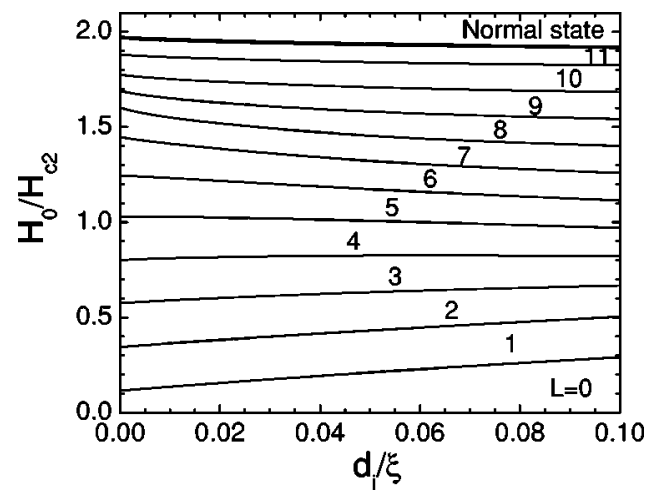

FIG. 11. Phase diagram: the ground state transition field $H_{0} / H_{c 2}$ as a function of the blind hole thickness $d_{i}$ for a superconducting sample with radius $R_{0}=4.0 \xi$, thickness $d=0.1 \xi, \kappa=1.0$, and with blind hole radius $R_{i}=2.0 \xi$. 


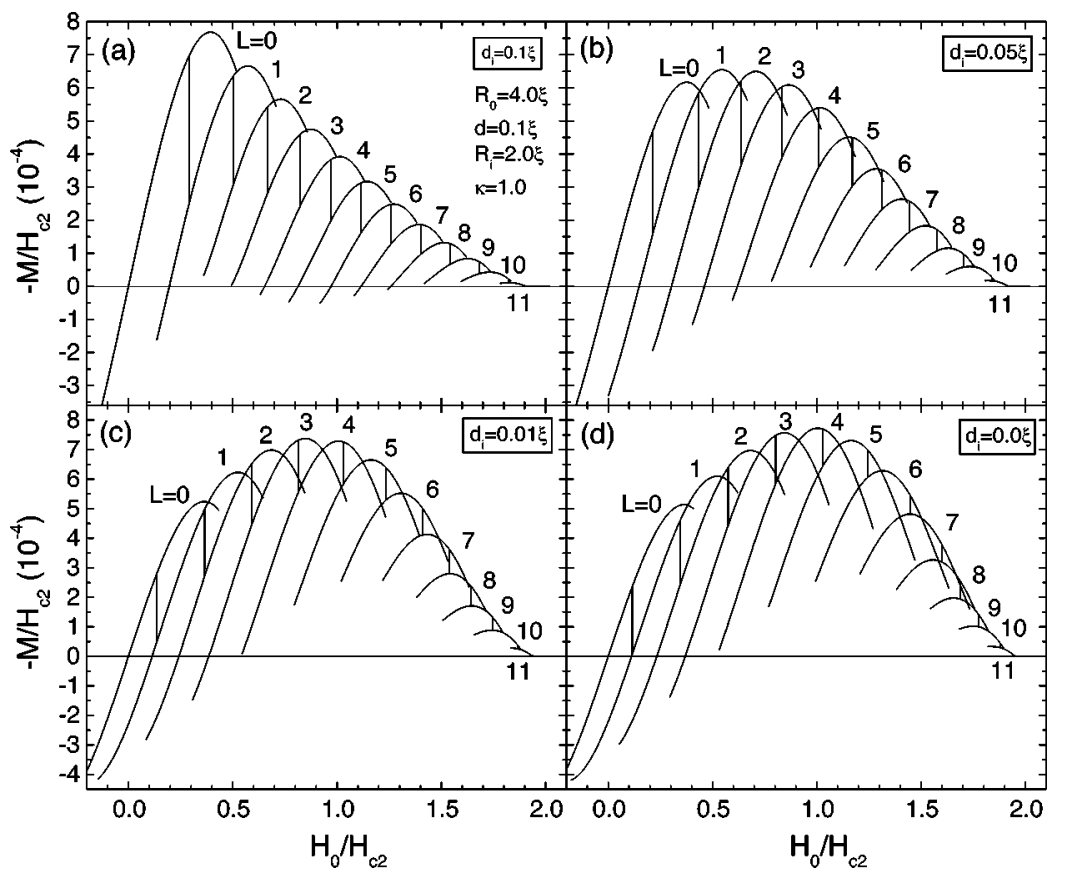

magnetic field. This field is $H_{c 3} / H_{c 2}=1.91$ for the disk and $H_{c 3} / H_{c 2}=1.92$ for the disk with the blind hole of thickness $d_{i}=0.05 \xi$. In this case less states are able to nucleate into a multivortex state, namely $L=2,3$, and 4 . Moreover, the magnetic field region, over which we found the multivortices, is also decreased. When we decrease the thickness of the blind hole [Fig. 10(c)] the vortex states become more stable and the ground state transitions occur at lower fields for lower vorticity. Notice that now the $L=1$ state remains stable even for negative applied fields. The $S / N$ transition field now is $H_{c 3} / H_{c 2}=1.95$. In this case all states are giant vortex states. By further decreasing the blind hole thickness the free energy approaches the energy of the superconducting ring [Fig. $10(\mathrm{~d})]$. The $S / N$ transition field of the ring is the highest one and equals $1.96 H_{c 2}$, which can also be seen from the phase diagram in Fig. 11. The phase diagram shows that for the states with lower vorticity $(L \leqslant 4)$ the ground state transition fields are higher for thicker blind holes, i.e., larger $d_{i}$, while for states with larger vorticity $(L>4)$ the ground state transition fields increase with decreasing $d_{i}$. From Figs. 6, 9, and 11 it is obvious that, regardless on the size of the disk and the blind hole, the thinner blind hole favors penetration of the first vortex in the sample (lower applied field necessary), due to enhanced compression of the field in the blind hole. However, the behavior of the critical field for penetration of the following vortices is determined by a competition between the pinning effects of the blind hole and repulsion between the vortices in the sample. In the case of a larger hole shown in Fig. 11, the pinning effects dominate till the fifth vortex penetrates the blind hole, reinforcing the repulsion between vortices. For $L>4$ vorticity, it is more energetically favorable that the vortices sit further from each other, when the confinement effects of the blind hole are weaker (larger $d_{i}$ ), resulting in a decrease of the threshold applied flux as function of the thickness of the blind hole.

Figures 12(a)-12(d) show the magnetization for the superconducting samples of Figs. 10(a)-10(d). The magnetization is calculated using Eq. (10) after averaging the field only over the superconductor, namely excluding the hole in the superconducting ring case. In these figures the vertical lines indicate the ground state transitions (see Fig. 10). In the case of the superconducting disk [Fig. 12(a)] the maxima in the magnetization curve decrease with increasing vorticity $L$, since most of the applied flux is expelled from the superconductor in the Meissner state. For the given value of the GL parameter the states with $L=1,4,5,6,7$, and 8 show partly paramagnetic response (i.e., $-M<0$ ). Notice that the insertion of a blind hole enhances such a paramagnetic response [Figs. 12(b) and 12(c)] for the small $L$-states. The largest amplitude of the magnetization is found for the $L=1$ state. When we decrease the thickness of the blind hole [Fig. 12(c)] (i) the maximum in the magnetization shifts to higher vorticity, (ii) less states show paramagnetic response, and (iii) the magnetization approaches the one of the superconducting ring case [Fig. 12(d)], which is maximal for the $L$ $=4$ state. The magnetization of the superconducting ring, when the field is averaged over the whole $R_{0}$ area, i.e., including the hole (Fig. 13) shows qualitatively similar features as the magnetization of the superconducting disk; but in this case the value of the magnetization is larger, the paramagnetic response is larger, and the magnetization value at the thermodynamic transition fields increases with $L$ up to $L=3$.

Figures 14(a) and 14(b) show the magnetic-field range $\Delta H_{s}=H_{\text {penetration }}-H_{\text {expulsion }}$ over which the vortex state with vorticity $L$ is stable and the magnetic-field range $\Delta H_{g}$ over which the given vortex state is the ground state. For the disk case, the results are given by full circles (solid line), for the disk with the blind hole with thickness $d_{i}=0.05 \xi$ by open circles (dashed line), $d_{i}=0.01 \xi$ by full squares (dash-dotted line) and for the superconducting ring by open squares (dotted line). Notice that in each case the Meissner state, i.e., the $L=0$ state, has the largest stability region. For the homogeneous disk $\Delta H_{s}$ exhibits a local maximum at $L=4$. The 


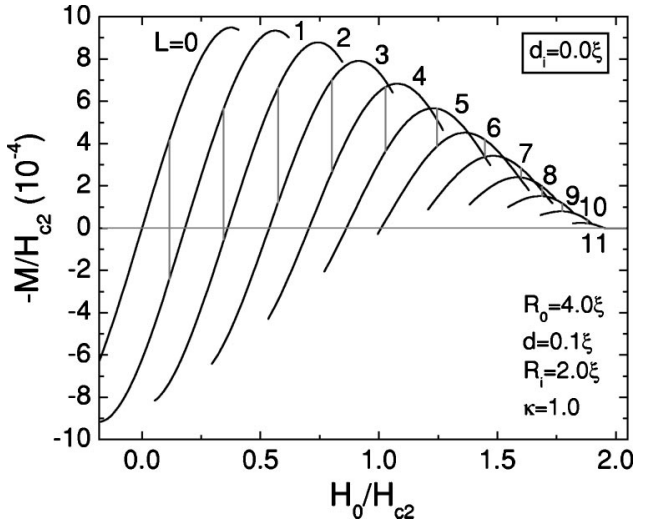

FIG. 13. The magnetization of the superconducting ring with outer radius $R_{0}=4.0 \xi$, thickness $d=0.1 \xi, \kappa=1.0$, and inner radius $R_{i}=2.0 \xi$, when the magnetic field is averaged over an area of radius $R_{0}$.

Meissner state becomes less stable when the blind hole is present. The transition to the $L=1$ state occurs at lower magnetic field, indicating that the presence of the blind hole significantly facilitates the penetration of the first vortex in the sample. The lowest stability region of the $L=0$ state is found for the superconducting ring. With decreasing the thickness of the blind hole the stability region of the vortex states with $L<7$ increases, but for $L>7$ the stability region $\Delta H_{s}$ is almost independent of $d_{i}$, which is a consequence of the fact that for large $L$-values superconductivity is destroyed in the

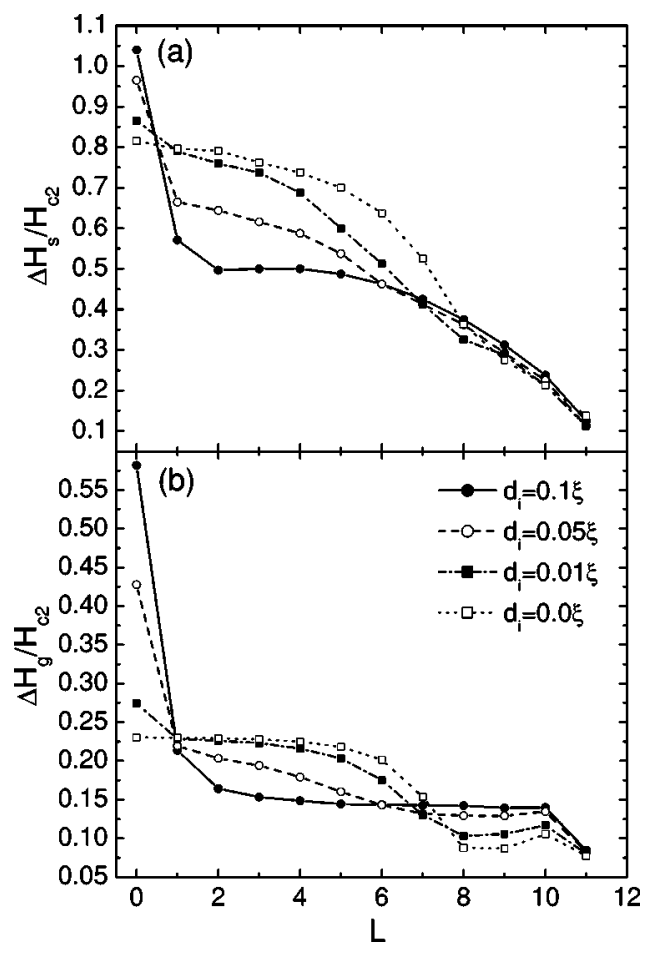

FIG. 14. (a) The magnetic-field range $\Delta H_{s}$ over which the vortex state with vorticity $L$ is stable and (b) the magnetic-field range $\Delta H_{g}$ over which the given vortex state is the ground state, as function of the vorticity $L$. The sample parameters are the same as in Fig. 10.

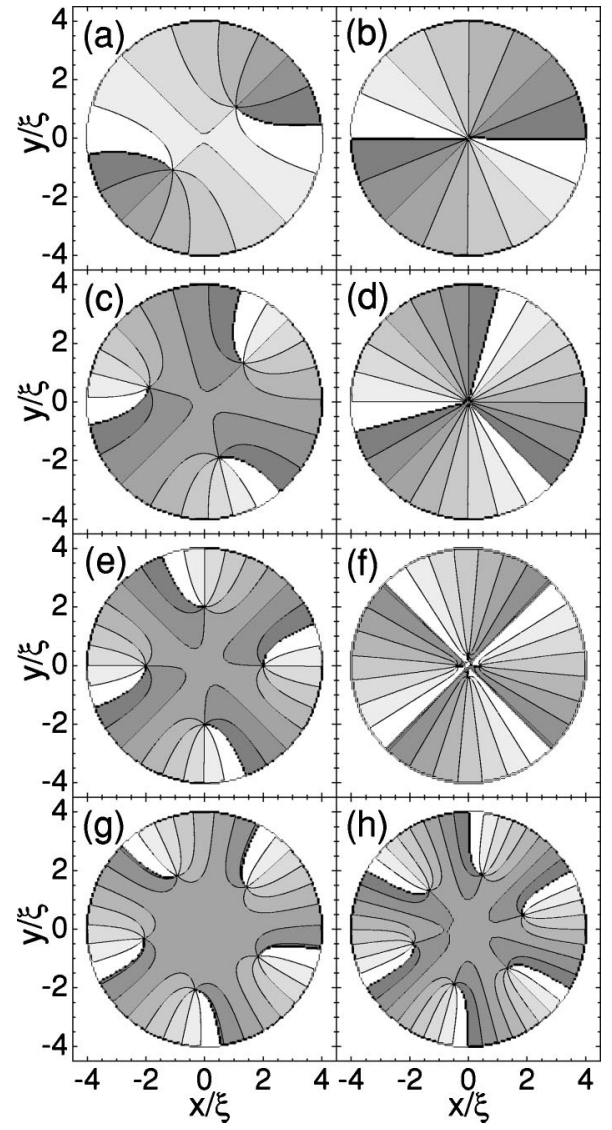

FIG. 15. Contour plot of the phase of the order parameter for the superconducting disk with radius $R_{0}=4.0 \xi$, thickness $d=0.1 \xi$ and $\kappa=1.0$ for the vortex states with $L=2$ (a) and (b), 3 (c) and (d), 4 (e) and (f), $5(\mathrm{~g})$, and $6(\mathrm{~h})$ at $H_{0} / H_{c 2}=0.36$ and $0.55,0.5$ and 0.77 , 0.64 and $0.87,0.77,0.92$, respectively. Phases near zero are given by white regions, phases near $2 \pi$ by dark gray regions.

center of the disk and consequently it does not matter whether or not a blind hole is present in that region of the disk. A similar tendency is observed for the ground state magnetic field range $\Delta H_{g}(L)$ [Fig. 14(b)] with the exception that for $L>7 \Delta H_{g}(L)$ decreases slightly with decreasing $d_{i}$. A similar tendency is seen for $\Delta H_{s}$ but to a smaller extent.

As we have shown before, many of the important features connected with the pinning of vortices by holes in the superconductors can be reproduced if thin blind holes are used instead. However, in the case of the superconducting rings, the flux is compressed in the hole and this quasigiant vortex can be recognized only by the phase of the order parameter in the superconductor around the hole. The advantage of blind holes lies in the fact that the real vortex structure inside the pinning center can be visualized. In what follows, we investigate the influence of the thickness of the blind hole on the vortex configurations. As was shown in Ref. 29, in superconducting disks transitions from the multivortex state to the giant vortex state can occur. In our disk sample [see Fig. 10(a)] such transitions appear for the states with $L=2,3,4,5$, and 6 . The number of multivortex states decreases with decreasing blind hole thickness. Figures 15(a)-15(h) show the phase of the order parameter of the disk for the states with $L=2-6$. Phases near zero are given by white regions and 


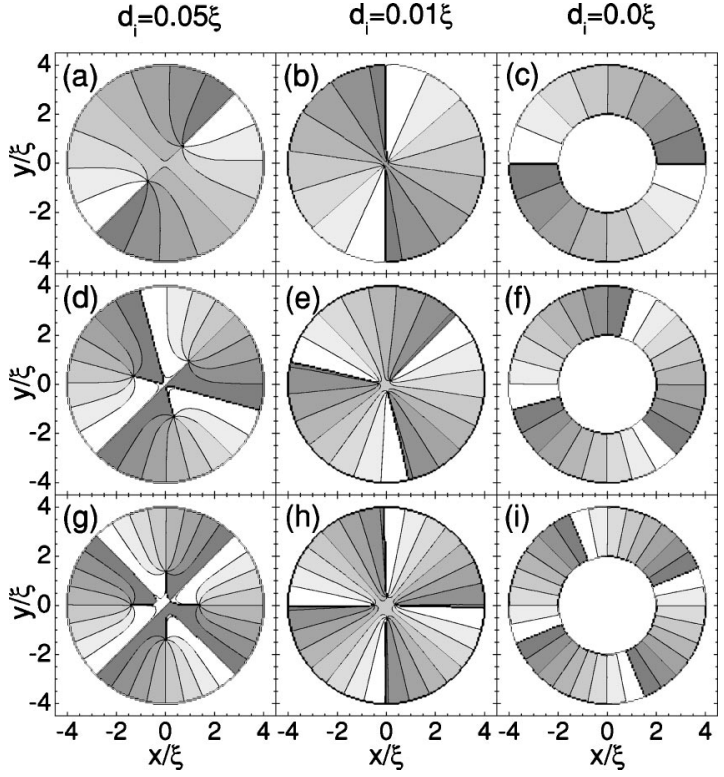

FIG. 16. Contour plot of the phase of the order parameter for a superconducting disk with radius $R_{0}=4.0 \xi$, thickness $d=0.1 \xi, \kappa$ $=1.0$ for blind holes with radius $R_{i}=2.0 \xi$ and thickness $d_{i}=0.05 \xi$ (a,d,g), $d_{i}=0.01 \xi(\mathrm{b}, \mathrm{e}, \mathrm{h})$ and $d_{i}=0.0 \xi(\mathrm{c}, \mathrm{f}, \mathrm{i})$ for the states with $L$ $=2(\mathrm{a}, \mathrm{b}, \mathrm{c}), 3(\mathrm{~d}, \mathrm{e}, \mathrm{f})$, and $4(\mathrm{~g}, \mathrm{~h}, \mathrm{i})$, at $H_{0} / H_{c 2}=0.22,0.41$, and 0.6 , respectively. Phases near zero are given by white regions, phases near $2 \pi$ by dark gray regions.

phases near $2 \pi$ by dark gray regions. For the $L=2$ state, in lower magnetic fields two individual vortices are separated from each other [Fig. 15(a)] and at the field $H_{0} / H_{c 2}=0.55$ they join into one giant vortex [Fig. 15(b)]. The multivortex to the giant vortex state transition for the $L=3$ state occurs at $H_{0} / H_{c 2}=0.77$ [Fig. 15(d)]. The $L=4$ state shows the "traditional" multivortex structure [Fig. 15(e)] where all vortices sit at the corners of the polygon. The multivortex to giant vortex transition occurs at $H_{0} / H_{c 2}=0.87$ [Fig. 15(f)]. Analogously, in small magnetic fields the states with $L=5$ and $L$ $=6$ show ring vortex structure [Figs. $15(\mathrm{~g})$ and $15(\mathrm{~h})$ ], which are recently of large scientific interest (see Refs. 30 and 31), but with increasing the applied magnetic field vortices move to the center and form one giant vortex. The states with even higher vorticity are giant vortex states.

The arrangements of vortices in samples containing a blind hole in the center is shown in Fig. 16 by the phase of the order parameter for the states with lower vorticity ( $L$ $\leqslant 4)$. When the blind hole with thickness $d_{i}=0.05 \xi$ and radius $R_{i}=2.0 \xi$ is present in the superconducting disk with $d$ $=0.1 \xi$ and $R_{0}=4.0 \xi$, the two vortices are closer to each other [Fig. 16(a)] than in the disk case. With decreasing the thickness of the blind hole these two vortices come closer to each other [Fig. 16(b)] and form a giant vortex with vorticity $L$ $=2$. Obviously, when the blind hole is present in the sample vortices are pinned by the hole, and therefore located closer to the center for all vortex states as compared to the disk case (Fig. 15). With decreasing the thickness of the hole the vortices are compressed more to the central region of the sample. In the case of the superconducting ring it is not possible to see the vortex structure [Figs. 16(c), 16(f), and 16(i)].

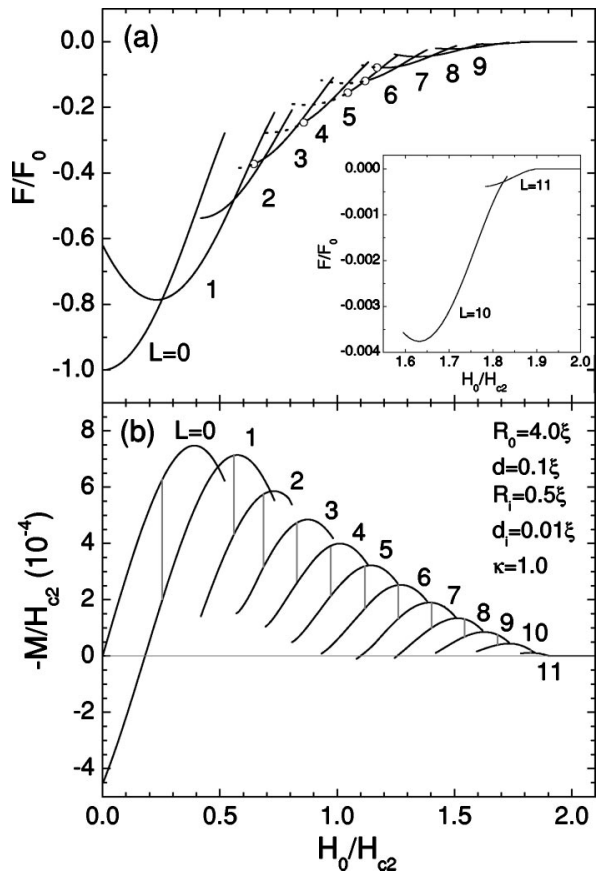

FIG. 17. The free energy (a) and the magnetization (b) as a function of the applied magnetic field of a disk with radius $R_{0}$ $=4.0 \xi$ and thickness $d=0.1 \xi$ for a blind hole in the center with radius $R_{i}=0.5 \xi$ and thickness $d_{i}=0.01 \xi$. The GL parameter $\kappa=1.0$. The inset shows the free energy for higher vorticity and vertical gray lines indicate ground state transitions. The multivortex states are plotted by dotted curves and the transitions from the multivortex state to the giant vortex state are indicated by open circles.

In the above samples, the radius of the blind hole $R_{i}$ is large enough and all vortices are individually located inside the blind hole forming the multivortex state, giant vortex state, or combination of them. Next we consider a superconducting disk with a small radius blind hole in the center. Figures 17(a) and 17(b) show the free energy and magnetization of the superconducting disk with radius $R_{0}=4.0 \xi$, thickness $d_{0}=0.1 \xi$ and containing a blind hole with radius $R_{i}=0.5 \xi$ and thickness $d_{i}=0.01 \xi$. The inset shows the enlargement of the free energy for the states with higher vorticity and vertical gray lines in the magnetization [see Fig. 17(b)] indicate the ground state transitions. The multivortex states are plotted by dotted curves and the transitions from the multivortex state to the giant vortex state are indicated by open circles. In this case vortex states up to $L=11$ can nucleate with a $S / N$ state transition field $H_{0} / H_{c 2}=1.91$. The states with vorticity $L=3-7$ are multivortex states. The $L=1$ state is stable over a larger magnetic-field range than the other states.

Figures 18(a)-18(h) show the phase of the order parameter of the sample for the states with $L=3-7$ at the different values of the applied magnetic field. For the $L=2$ state vortices are close to each other and located in the blind hole, as in the case of samples with larger blind holes. But for the $L=3$ state one vortex is inside the blind hole and the other are outside of it [Fig. 18(a)]. By further increasing the field the vortices move to the center [Fig. 18(b)]. For the $L=4,5$, 6 states one vortex is in the hole and others make a triangu- 


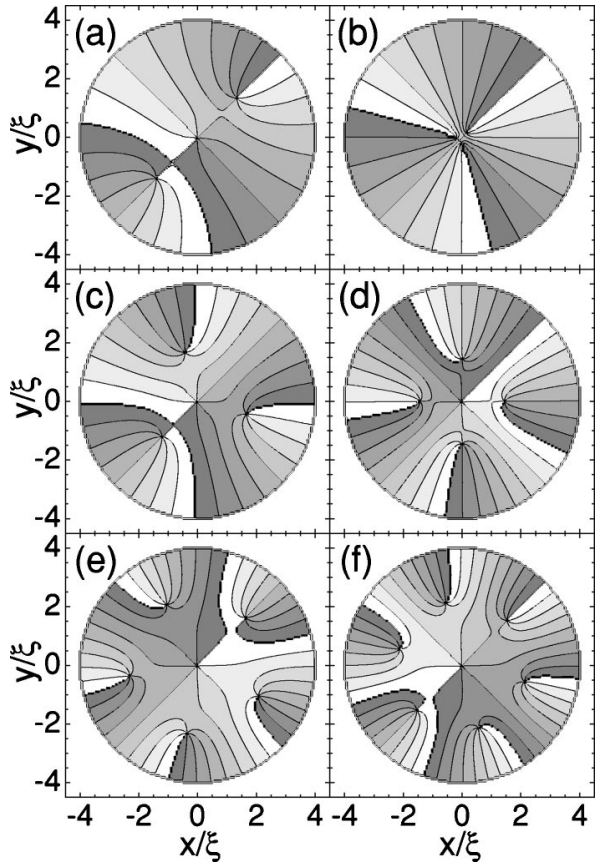

FIG. 18. The phase of the order parameter for the superconducting disk with radius $R_{0}=4.0 \xi$, thickness $d=0.1 \xi, \kappa=1.0$ for a blind hole in the center with radius $R_{i}=0.5 \xi$ and thickness $d_{i}=0.01 \xi$ for the vortex states with $L=3$ (a) and (b), 4 (c), 5 (d), 6 (e) and (f), and $7(\mathrm{~g})$ and $(\mathrm{h})$ at $H_{0} / H_{c 2}=0.57,0.65,0.80,1.0,0.94$, and 1.1 , respectively. Phases near zero are given by white regions, phases near $2 \pi$ by dark gray regions.

lar, square and "pentagonal" lattice around the hole [Figs. 18(c)-18(e)]. At small fields the state with $L=7$ makes a "shell" structure ${ }^{30,31}$ with one vortex in the center [Fig. 18(f)]. By increasing the magnetic field these vortices move toward the hole and form a giant vortex.

\section{NONCENTRAL LOCATION OF THE BLIND HOLE}

Until now we considered only cylindrically symmetric superconductors. In the next step we investigate the nonsymmetric case, i.e., when the blind hole is shifted from the center of the sample over a distance $a$. As an example we take a superconducting disk with radius $R_{0}=4.0 \xi$, thickness $d=0.1 \xi$ with a blind hole with radius $R_{i}=1.0 \xi$ and thickness $d_{i}=0.01 \xi$ moved over a distance $a=2.0 \xi$ in the $-y$-direction. Figures 19(c) and 19(d) show the free energy and magnetization of this sample. To compare with the symmetrical case, we also plotted the free energy and magnetization for the superconductor with a blind hole in the center [Figs. 19(a) and 19(b)]. In the latter case $L=11$ vortices can be captured into the superconductor while it transits to the normal state at $H_{0} / H_{c 2}=1.91$. The breaking of the symmetry changes the superconducting state considerably. In this case the maximal number of vortices in the sample is $L=15$ and the stability of the states with lower vorticity are decreased. The ground state free energy of the nonsymmetric sample is lower for the states with $L>3$, which leads to a higher critical field $H_{c 3} / H_{c 2}=2.47$. It is noticeable that transitions between vortex states after $L=10$ occur without a jump in the magnetization.

Figures 20(a)-20(h) show the distribution of vortices in the samples by the phase of the order parameter and Cooperpair density. For the symmetric system, i.e., $a=0$, we found only giant vortex states. Figures 20(a) and 20(b) show the Cooper-pair density and the phase of the order parameter for the $L=2$ state in that sample. By breaking the circular symmetry of the system, multivortex states are stabilized. Figures 20(c)-20(g) show the Cooper-pair density of such multivortex states. The vortex nucleated at the blind hole is for $L \geqslant 5$ a giant vortex with vorticity 2 [see, e.g., Fig. 20(h)].

\section{VII. $H-T$ PHASE DIAGRAM}

So far, our calculations have been done for fixed temperature $T$. Now we will include temperature in our numerical

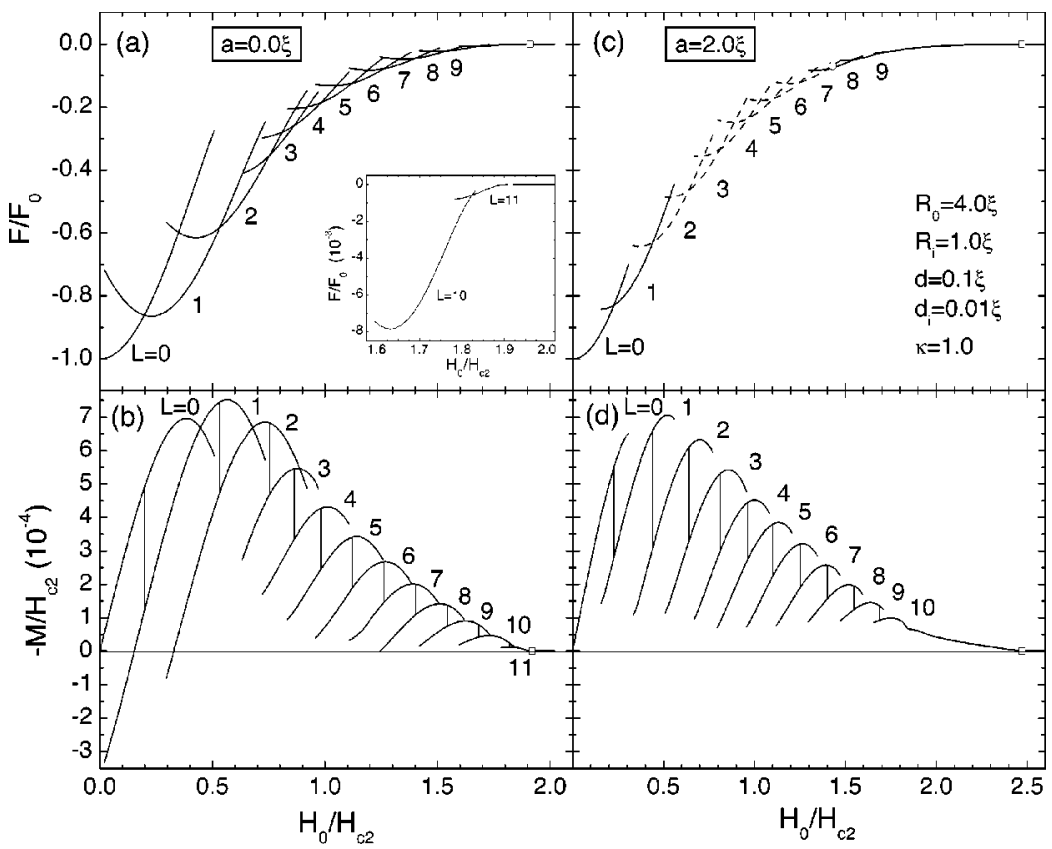

FIG. 19. The free energy and magnetization as a function of the applied magnetic field of the superconducting disk with radius $R_{0}=4.0 \xi$, thickness $d=0.1 \xi$ with a blind hole with radius $R_{i}=1.0 \xi$, and thickness $d_{i}=0.01 \xi$, when the blind hole is in the center (a) and (b) and moved over a distance $a$ $=2.0 \xi$ in the $-y$ direction (c) and (d). The multivortex states are plotted by dashed curves and the transition from the multivortex state to the giant vortex state is indicated by open circle in (c). Open squares show the $S / N$ transition fields. 


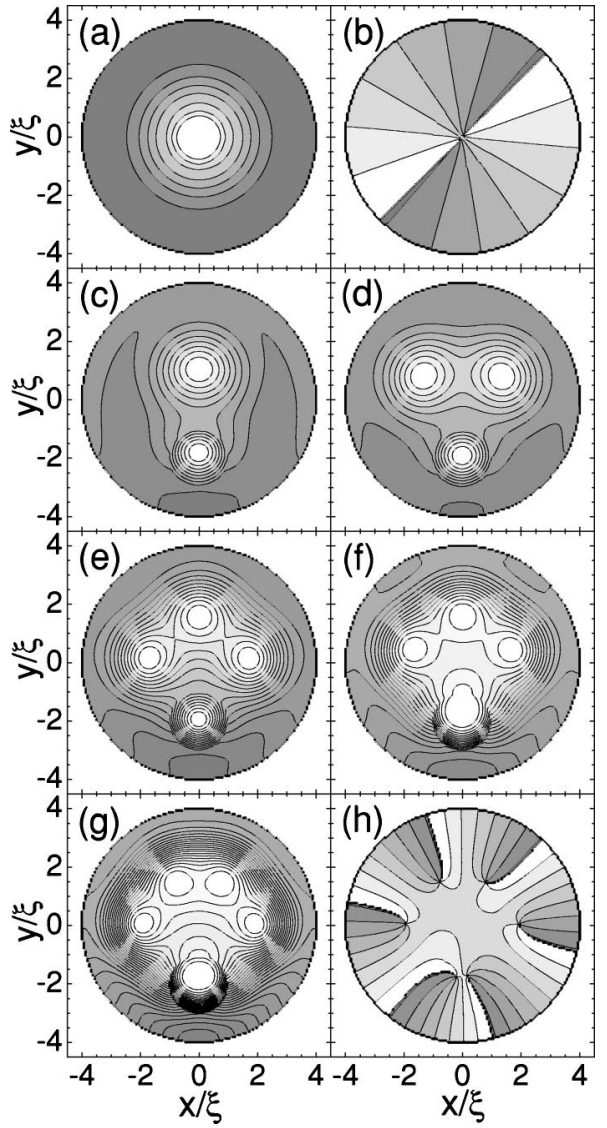

FIG. 20. Contour plot of the Cooper-pair density and phase of the order parameter (b) and (h) for the superconducting disk with radius $R_{0}=4.0 \xi$, thickness $d=0.1 \xi$ with a blind hole with radius $R_{i}=1.0 \xi$ and thickness $d_{i}=0.01 \xi$, when the blind hole is in the center (a) and (b) and moved over a distance $a=2.0 \xi$ in the $y$ direction (c)-(h), corresponding to the vortex states with $L=2$ (a) and (c), 3 (d), 4 (e), 5 (f), and 6 (g) at $H_{0} / H_{c 2}=0.55$ and $0.65,0.82,1.0$, and 1.12 , respectively.

calculations through the temperature dependence of the coherence length [see Eqs. (11) and (12)]. Therefore, all distances will be expressed in units of $\xi(0)$, magnetic field in $H_{c 2}(0)$, and temperature in units of the zero-magnetic-field critical temperature $T_{c 0}$. We consider two samples, namely the superconducting disk with radius $R_{0}=1.5 \mu \mathrm{m}$ and thickness $d=100 \mathrm{~nm}$, with and without a blind hole with radius $R_{i}=0.75 \mu \mathrm{m}$ and thickness $d_{i}=10 \mathrm{~nm}$. We choose the coherence length $\xi(0)=120 \mathrm{~nm}$ and the penetration depth $\lambda(0)$ $=140 \mathrm{~nm}$, which are typical experimental values for lowtemperature mesoscopic superconductors.

The $H-T$ phase diagram is shown in Figs. 21(a)-21(c) for the disk (a), the disk with a blind hole (b), and for the superconducting ring (c) for the states with vorticity up to $L=5$. In the presence of the blind holes vortices enter the sample at higher temperatures and these states have a larger stability region compared to the case of the disk (except for the Meissner state where the opposite tendency is noticed). The $S / N$ transition field at fixed temperature and the critical temperature at the given field is higher for the sample with the blind hole. For values of the parameters used here, the critical field is increased more than $20 \%$ and the critical tempera-

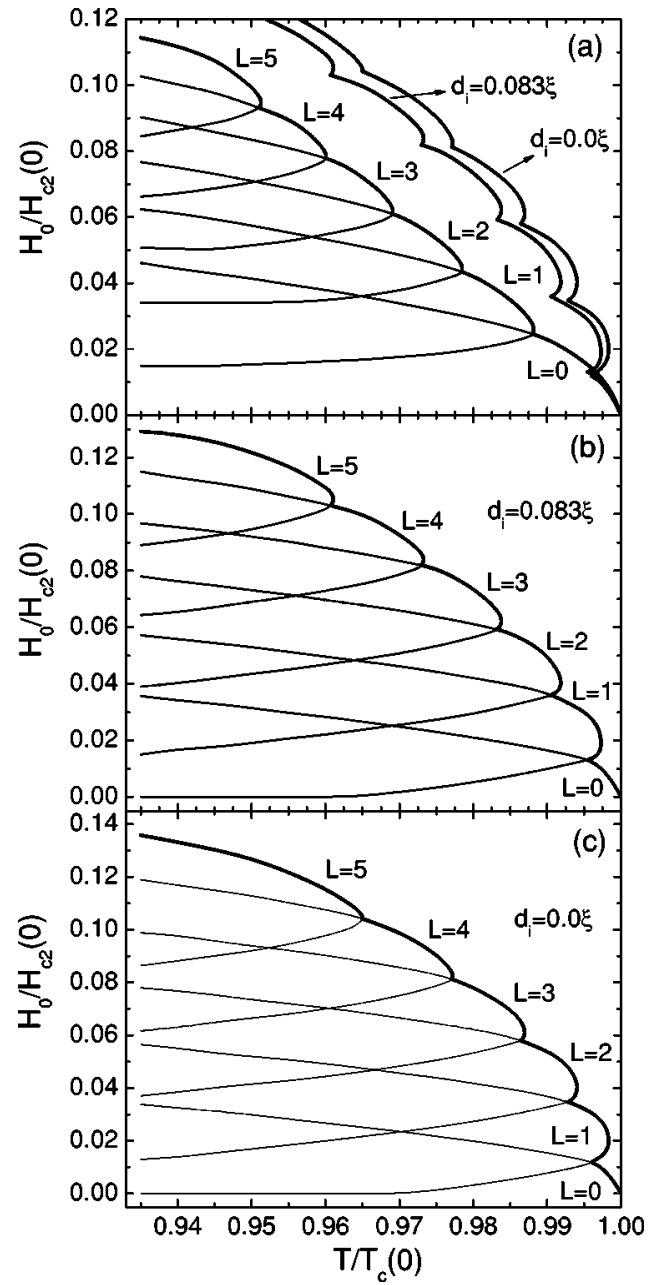

FIG. 21. The $H-T$ phase diagram and the stability area for the states with vorticity up to $L=5$ for (a) the disk, (b) the disk with blind hole, and (c) the superconducting ring. The radius of the disk is $R_{0}=12.5 \xi(0)$, the thickness of the disk $d=0.83 \xi(0)$, the radius of the blind hole $R_{i}=6.25 \xi(0)$, the thickness of the blind hole $d_{i}$ $=0.083 \xi(0)$, and the GL parameter $\kappa$ equals $1.167(\xi(0)=120 \mathrm{~nm})$. Thick solid curves indicate the superconducting/normal transitions.

ture by $\sim 1.5 \%$. Therefore, introduction of the blind hole in the superconducting sample is a powerful tool for enhancement of the critical parameters. The critical parameters of the sample with a blind hole are close to the parameters of the superconducting ring.

\section{CONCLUSIONS}

We studied the nucleation of superconductivity in superconducting disks with a blind hole in the center of the sample. The influence of the sample shape on the superconducting state was thoroughly investigated. We found that the increase of the steepness of the edges of the blind-hole-like cavity in the superconductor leads to a shift of the $S / N$ transition field to higher magnetic fields, but the maximal number of vortices remains the same. The Cooper-pair density, magnetic field and current density distributions show that the flux trapped in the superconductor is compressed more into 
the center of the sample, when the thickness of the sample changes more steeply.

We also investigated superconducting disks with a perfect blind hole in the center in the case of relatively small disks $(R=2.0 \xi)$. When the radius of the blind hole is much smaller than the radius of the disk, the maximal number of allowed vortices is the same for all considered samples, regardless of the thickness of the blind hole. On the other hand, the decrease of the blind-hole thickness leads to higher $S / N$ transition fields, and the free energy of the sample with blind hole approaches the energy of the superconducting ring. The local magnetic-field distribution shows that for the $L=1$ state, the magnetic field in the center of the sample for the superconducting ring is lower than at the blind hole edge. For the sample with the blind hole the magnetic field is maximal in the center, showing one of the significant differences between the hole and a blind hole as pinning centers. For the larger radii of the blind hole the maximal number of vortices in the sample increases with decreasing the thickness of the blind hole and approaches to the number of vortices in the case of he superconducting ring.

In order to investigate the vortex configurations inside the blind hole (as opposed to the hole as a pinning center), we considered superconducting samples with larger sizes. For the value of the GL parameter $\kappa=1.0$ the states with $L \leqslant 6$ show multivortex states and a further increase of the applied magnetic field leads to the giant vortex state. When the blind hole is included into the disk the free energy of all vortex states lowers and the $S / N$ state transition occurs at higher magnetic field. A variety of vortex configurations is possible in the blind hole, since the vortices are confined to the hole. In this case, less vortex states form a multivortex state and the range of the magnetic field at which multivortex states are present is also decreased for fixed $L$. A thinner blind hole leads to more stable vortex states and ground state transitions between different vortex states occur at lower fields. The states with higher vorticity illustrate stronger flux expulsion in samples with a blind hole as compared to the pure disk case. For a small radius of the blind hole a limited number of vortices are situated in the hole and other vortices are located in the superconducting region forming a "shell" vortex structure. In this case, the shell "magic numbers" can effectively be controlled by the ratio between the size of the disk and its blind hole. We also considered the nonsymmetric case when the blind hole is moved over some distance from the center of the sample. In this case the maximal number of vortices in the sample is increased, the stability of these states is decreased, and multivortex states are favorable.

The $H-T$ phase diagram calculated for the case of the disk and the disk with a blind hole shows that the critical field at a given temperature and the critical temperature at fixed field are higher for samples with a blind hole, and their values approach the phase boundary of the superconducting ring.

\section{ACKNOWLEDGMENTS}

This work was supported by the Flemish Science Foundation (FWO-Vl), the Belgian Science Policy, the "Onderzoeksraad van de Universiteit Antwerpen" (GOA), and the ESF program on "Vortex matter." B.J.B. acknowledges support from FWO-Vl and the Japanese Society for the Promotion of Science.
*Electronic address: golibjon.berdiyorov@ua.ac.be

†resent address: Institute of Materials Science, University of Tsukuba, 1-1-1, Tennoudai, Tsukuba 305-8573, Japan.

†Electronic address: francois.peeters@ua.ac.be

${ }^{1}$ O. Buisson, P. Gandit, R. Rammel, Y. Y. Wang, and B. Pannetier, Phys. Lett. A 150, 36 (1990).

${ }^{2}$ A. K. Geim, I. V. Grigorieva, S. V. Dubonos, J. G. S. Lok, J. C. Maan, A. E. Filippov, and F. M. Peeters, Nature (London) 390, 256 (1997).

${ }^{3}$ A. K. Geim, S. V. Dubonos, J. G. S. Lok, I. V. Grigorieva, J. C. Maan, L. Theil Hansen, and P. E. Lindelof, Appl. Phys. Lett. 71, 2379 (1997).

${ }^{4}$ P. S. Deo, V. A. Schweigert, F. M. Peeters, and A. K. Geim, Phys. Rev. Lett. 79, 4653 (1997).

${ }^{5}$ A. K. Geim, S .V. Dubonos, J. G. S. Lok, M. Henini, and J. C. Maan, Nature (London) 396, 144 (1998).

${ }^{6}$ A. K. Geim, S. V. Dubonos, I. V. Grigorieva, K. S. Novoselov, F. M. Peeters, and V. A. Schweigert, Nature (London) 407, 55 (2000).

${ }^{7}$ F. M. Peeters, V. A. Schweigert, and B. J. Baelus, Physica C 369, 158 (2002).

${ }^{8}$ V. A. Schweigert and F. M. Peeters, Phys. Rev. B 57, 13817 (1998).
${ }^{9}$ V. A. Schweigert, F. M. Peeters, and P. S. Deo, Phys. Rev. Lett. 81, 2783 (1998).

${ }^{10}$ P. S. Deo, F. M. Peeters, and V. A. Schweigert, Superlattices Microstruct. 25, 1195 (1999).

${ }^{11}$ J. J. Palacios, Phys. Rev. B 58, R5948 (1998); E. Akkermans and K. Mallick, J. Phys. A 32, 7133 (1999).

${ }^{12}$ W. A. Little and R. D. Parks, Phys. Rev. Lett. 9, 9 (1962); Phys. Rev. 133, A97 (1964).

${ }^{13}$ J. Berger and J. Rubinstein, Phys. Rev. Lett. 75, 320 (1995); Phys. Rev. B 56, 5124 (1997); ibid. 59, 8896 (1999).

${ }^{14}$ B. J. Baelus, F. M. Peeters, and V. A. Schweigert, Phys. Rev. B 61, 9734 (2000); 63, 144517 (2001).

${ }^{15}$ V. V. Moshchalkov, L. Gielen, C. Strunk, R. Jonckheere, X. G. Qiu, C. Van Haesendonck, and Y. Bruynseraede, Nature (London) 373, 319 (1995).

${ }^{16}$ V. V. Moshchalkov, X. G. Qiu, and V. Bruyndoncx, Phys. Rev. B 55, 11793 (1997).

${ }^{17}$ V. Bruyndoncx, J. G. Rodrigo, T. Puig, L. Van Look, V. V. Moshchalkov, and R. Jonckheere, Phys. Rev. B 60, 4285 (1999).

${ }^{18}$ F. M. Peeters and X. Q. Li, Appl. Phys. Lett. 72, 572 (1998).

${ }^{19}$ C. P. Herring, Phys. Lett. 47A, 105 (1974).

${ }^{20}$ A. Bezryadin and B. Pannetier, J. Low Temp. Phys. 102, 73 (1996). 
${ }^{21}$ J. E. Bonevich, K. Harada, T. Matsuda, H. Kasai, T. Yoshida, G. Pozzi, and A. Tonomura, Phys. Rev. Lett. 70, 2952 (1993).

${ }^{22}$ S. Behler, S. H. Pan, P. Jess, A. Baratoff, H. J. Guntherodt, F. Levy, G. Wirth, and J. Wiesner, Phys. Rev. Lett. 72, 1750 (1994).

${ }^{23}$ K. Harada, O. Kamimura, H. Kasai, T. Matsuda, A. Tonomura, and V. V Moshchalkov, Science 274, 1167 (1996).

${ }^{24}$ S. Raedts, A. V. Silhanek, M. J. Van Bael and V. V Moshchalkov, Phys. Mag. 25, 209 (2003); Physica C 404, 298 (2004).

${ }^{25}$ A. Bezryadin, Yu. N. Ovchinnikov, and B. Pannetier, Phys. Rev. B 53, 8553 (1995).
${ }^{26}$ A. Buzdin and M. Daumens, Physica C 332, 108 (2000).

${ }^{27}$ V. V. Schmidt, P. Müller, and A. V. Ustinov, The Physics of Superconductors: Introduction to Fundamentals and Applications (Springer-Verlag, Berlin, 1997).

${ }^{28}$ Qiang Du, Max D. Gunzburger, and J. S. Peterson, Phys. Rev. B 51, 16194 (1995).

${ }^{29}$ B. J. Baelus and F. M. Peeters, Phys. Rev. B 65, 104515 (2002).

${ }^{30}$ M. V. Milošević and F. M. Peeters, Phys. Rev. B 68, 024509 (2003).

${ }^{31}$ B. J. Baelus, L. R. E. Cabral, and F. M. Peeters, Phys. Rev. B 69, 064506 (2004). 